\title{
A DOLBEAULT-GROTHENDIECK LEMMA ON COMPLEX SPACES VIA KOPPELMAN FORMULAS
}

\author{
MATS ANDERSSON \& HÅKAN SAMUELSSON
}

\begin{abstract}
Let $X$ be a complex space of pure dimension. We introduce fine sheaves $\mathscr{A}_{q}^{X}$ of $(0, q)$-currents, which coincides with the sheaves of smooth forms on the regular part of $X$, so that the associated Dolbeault complex yields a resolution of the structure sheaf $\mathscr{O}^{X}$. Our construction is based on intrinsic and quite explicit semi-global Koppelman formulas.
\end{abstract}

\section{INTRODUCTION}

The fundamental Dolbeault-Grothendieck lemma assures that a smooth $\bar{\partial}$-closed $(0, q)$-form on a complex $n$-dimensional manifold $X$ is locally $\bar{\partial}$-exact if $q \geq 1$ and holomorphic if $q=0$. This means precisely that the sheaf complex

$$
0 \rightarrow \mathscr{O}^{X} \hookrightarrow \mathscr{E}_{0,0}^{X} \stackrel{\bar{\partial}}{\longrightarrow} \mathscr{E}_{0,1}^{X} \stackrel{\bar{\partial}}{\longrightarrow} \cdots \stackrel{\bar{\partial}}{\longrightarrow} \mathscr{E}_{0, n}^{X} \rightarrow 0
$$

is exact, where $\mathscr{E}_{p, *}^{X}$ denote the fine sheaves of smooth $(p, *)$-forms on $X$; thus (1.1) the Dolbeault complex - is a fine resolution of the structure sheaf $\mathscr{O}^{X}$. That a sheaf is fine means that it is a module over $\mathscr{E}_{0,0}^{X}$ and thus partitions of unity are possible. If $E \rightarrow X$ is a holomorphic vector bundle and $\mathscr{O}(E)$ is the associated locally free $\mathscr{O}^{X}$ module we then get, by the abstract de Rham theorem, the generalized DolbeaultGrothendieck isomorphism, i.e., the representation of the sheaf cohomology groups $H^{q}(X, \mathscr{O}(E))$ as the obstruction to global solvability of the $\bar{\partial}$-equation.

The main result of this paper is a generalization of the Dolbeault-Grothendieck lemma to reduced complex spaces. To achieve this we construct intrinsic quite explicit semi-global weighted Koppelman formulas on subvarieties $X \subset \Omega$ of pseudoconvex domains $\Omega \subset \mathbb{C}^{N}$.

Abstractly, one can always find fine resolutions of $\mathscr{O}^{X}$ as was realized by R. Godement and A. Grothendieck in the '50s and '60s, but these resolutions are naturally very abstract. More concrete Dolbeault resolutions of $\mathscr{O}^{X}$ were constructed by Ancona-Gaveau for certain complex spaces in [1, 2, 23] in particular, their sheaves coincide with $\mathscr{E}_{0, *}^{X}$ on the regular part of $X$. Ancona and Gaveau assume that $X$ is normal, that $X_{\text {sing }}$ is a smooth manifold, and that there exists a desingularization of $X$ with certain hypotheses on the exceptional divisor. Their sheaves of differential forms are then the differential forms on this desingularization specified by jet conditions along the exceptional divisor.

2000 Mathematics Subject Classification. 32A26, 32A27, 32B15, 32C30.

The first author was partially supported by a grant from the Swedish Research Council. The second author wishes to thank the Department of Mathematics, University of Oslo, where parts of his work was done. 
Let $X$ be a complex space of pure dimension $n$ and let $\mathscr{O}^{X}$ be the structure sheaf of (strongly) holomorphic functions. Locally, $X$ can be considered as a subvariety of a domain $\Omega \subset \mathbb{C}^{N}, i: X \hookrightarrow \Omega$, and then $\mathscr{O}^{X}=\mathscr{O}^{\Omega} / \mathcal{J}_{X}$, where $\mathcal{J}_{X}$ is the sheaf in $\Omega$ of holomorphic functions vanishing on $X$. In the same way we say that $\phi$ is a smooth $(p, q)$-form on $X, \phi \in \mathscr{E}_{p, q}(X)$, if given a local embedding, there is a smooth form $\Phi$ in a neighborhood in the ambient space such that $\phi=i^{*} \Phi$ on $X_{r e g}$. It is well-known that this defines an intrinsic sheaf $\mathscr{E}_{p, q}^{X}$ on $X$.

It was proved by Malgrange, [29], that if a smooth function $\phi \in \mathscr{E}_{0,0}(X)$ is $\bar{\partial}$ closed, i.e., holomorphic, on $X_{\text {reg }}$, then $\phi$ is indeed (strongly) holomorphic. If $\phi$ is a smooth $\bar{\partial}$-closed $(0, q)$-form, $q \geq 1$, on $X$ and $X$ is embedded as a reduced complete intersection in a pseudoconvex domain, then Henkin-Polyakov, [24, proved that there is a solution $u$ to $\bar{\partial} u=\phi$ on $X_{\text {reg }}$ (which is not Stein in general). It was an open question for long whether this holds in more generality. In [9] we proved 1 that if $\phi$ is a smooth $\bar{\partial}$-closed $(0, q)$-form on a Stein space then there is a solution to the $\bar{\partial} u=\phi$ on $X_{\text {reg }}$. The proof is based on Koppelman formulas on $X$, and the smooth solution to $\bar{\partial} u=\phi$ in $X_{\text {reg }}$ is given (semiglobally) by an intrinsic integral formula $u(z)=\mathscr{K} \phi(z)$, cf., Theorem 1.4 and (1.4) below. Similar, but non-intrinsic formulas were obtained by Henkin-Polyakov, [24], in the special case of a reduced, embedded complete intersection. In [4], Koppelman formulas of Bochner-Martinelli type are used but these do not produce solutions to the $\bar{\partial}$-equation. In the recent paper [25] the $\bar{\partial}$-equation is studied with integral formulas on a not necessarily reduced projective variety that is a (locally) complete intersection.

Example 1.1. Let $X$ be the germ of the curve at $0 \in \mathbb{C}^{2}$ defined by $t \mapsto\left(t^{3}, t^{7}+t^{8}\right)$. If $\phi=\bar{w} d \bar{z}=3\left(\bar{t}^{9}+\bar{t}^{10}\right) d \bar{t}$ than one can verify that there is no solution $\psi=f\left(t^{3}, t^{7}+t^{8}\right)$, with $f$ smooth, to $\bar{\partial} \psi=\phi$. See, e.g., [35] for other examples.

From the example it is clear that our solution $u=\mathscr{K} \phi$ cannot have a smooth extension to $X$. However, it turns out that it has a current extension to all of $X$. For the definition of currents on $X$, see Section 2, It turns out that the singularities of $u$ at $X_{\text {sing }}$ are quite "mild"; in particular we can multiply it with any smooth form and plug it into the integral operator $\mathscr{K}$ again so that the Koppelman formula still holds. The resulting currents can again be multiplied by a smooth form and plugged into the formulas, etc. The currents obtained in this way in a finite number of steps form sheaves $\mathscr{A}_{q}^{X}$; for the precise definition, see Section [7. Here is our main result.

Theorem 1.2. Let $X$ be a reduced complex space of pure dimension $n$. The sheaves $\mathscr{A}_{q}^{X}$ are fine sheaves of $(0, q)$-currents on $X$, they contain $\mathscr{E}_{0, q}^{X}$, and moreover

(i) $\mathscr{A}^{X}:=\bigoplus_{q} \mathscr{A}_{q}^{X}$ is a module over $\mathscr{E}_{0, *}^{X}$,

(ii) $\left.\mathscr{A}_{q}^{X}\right|_{X_{\text {reg }}}=\left.\mathscr{E}_{0, q}^{X}\right|_{X_{\text {reg }}}$,

(iii) the complex

$$
0 \rightarrow \mathscr{O}^{X} \hookrightarrow \mathscr{A}_{0}^{X} \stackrel{\bar{\partial}}{\longrightarrow} \mathscr{A}_{1}^{X} \stackrel{\bar{\partial}}{\longrightarrow} \cdots \stackrel{\bar{\partial}}{\longrightarrow} \mathscr{A}_{n}^{X} \rightarrow 0
$$

is exact.

By the abstract theorem of de Rham we immediately get the following

\footnotetext{
${ }^{1}$ This material is published in 10 .
} 
Corollary 1.3. Let $X$ be a reduced pure-dimensional complex space, let $E \rightarrow X$ be a holomorphic vector bundle, and let $\mathscr{O}(E)$ be the associated locally free $\mathscr{O}^{X}$-module. Then we have the representation of sheaf cohomology

$$
H^{q}(X, \mathscr{O}(E)) \simeq \frac{\left\{\varphi \in \mathscr{A}_{q}(X, E) ; \bar{\partial} \varphi=0\right\}}{\left\{\bar{\partial} u ; u \in \mathscr{A}_{q-1}(X, E)\right\}}, \quad q \geq 1 .
$$

The semiglobal Koppelman formulas from [9] hold for currents in $\mathscr{A}^{X}$.

Theorem 1.4. Let $X$ be an analytic subvariety of pure dimension of a pseudoconvex domain $\Omega \subset \mathbb{C}^{N}$; take any relatively compact subdomain $\Omega^{\prime} \Subset \Omega$ and put $X^{\prime}=X \cap \Omega^{\prime}$. There are integral operators $\mathscr{K}: \mathscr{A}_{q+1}(X) \rightarrow \mathscr{A}_{q}\left(X^{\prime}\right)$ and $\mathscr{P}: \mathscr{A}_{0}(X) \rightarrow \mathscr{O}\left(\Omega^{\prime}\right)$ such that

$$
\begin{gathered}
\phi(z)=\bar{\partial} \mathscr{K} \phi(z)+\mathscr{K}(\bar{\partial} \phi)(z), \quad z \in X^{\prime}, \phi \in \mathscr{A}_{q}(X), q \geq 1, \\
\phi(z)=\mathscr{K}(\bar{\partial} \phi)(z)+\mathscr{P} \phi(z), \quad z \in X^{\prime}, \phi \in \mathscr{A}_{0}(X) .
\end{gathered}
$$

The operators $\mathscr{K}$ and $\mathscr{P}$ are given as

$$
\mathscr{K} \phi(z)=\int_{\zeta} k(\zeta, z) \wedge \phi(\zeta), \quad \mathscr{P} \phi(z)=\int_{\zeta} p(\zeta, z) \wedge \phi(\zeta),
$$

where $k$ and $p$ are integral kernels on $X \times X^{\prime}$ and $X \times \Omega^{\prime}$ respectively. These kernels are locally integrable with respect to $\zeta$ on $X_{r e g}$ and the integrals are principal values at $X_{\text {sing }}$. If $\phi$ vanishes in a neighborhood of an arbitrary point $x \in X$, then $\mathscr{K} \phi$ is smooth at $x$.

Any meromorphic $(k, 0)$-form $\phi$ on $X$, defines a principal value current on $X$ and $\bar{\partial} \phi=0$ means that $\phi$ is in $\mathcal{B}_{k}^{X}$, i.e., holomorphic in the sense of Barlet-HenkinPassare, cf., Example 2.8 below. The sheaf $\mathcal{B}_{k}^{X}$ is in general larger than the sheaf of (strongly) holomorphic $(k, 0)$-forms. Let $\mathcal{W}_{0, q}^{X}$ be the sheaf of pseudomeromorphic $(0, q)$-currents $\mu$ with the so-called SEP, the standard extension property. The SEP means roughly speaking that $\mu$ is determined by its values on any dense Zariski open subset of $X$. For instance, $\mathcal{W}^{X}$ contains all semi-meromorphic forms as well as push-forwards of such forms under modifications and simple projections $X \times Y \rightarrow X$.

For currents in $\mathcal{W}_{0, *}^{X}$ we introduce a stronger notion for the $\bar{\partial}$-operator, denoted $\bar{\partial}_{X}$, such that the following holds: If $\phi \in \mathcal{W}_{0,0}(X)$ and $\bar{\partial}_{X} \phi=0$ then $\phi \in \mathscr{O}(X)$. If $\phi \in \mathcal{W}_{0, q}(X), q \geq 1$, and $X$ is Stein then there is a solution to the $\bar{\partial}$-equation on $X_{\text {reg }}$; cf. Proposition 1.5 below and the succeeding remarks. To explain the operator $\bar{\partial}_{X}$, let us for simplicity here assume that $X$ is Cohen-Macaulay and embedded in a pseudoconvex domain $\Omega \subset \mathbb{C}^{N}$. From a locally free resolution of $\mathscr{O}^{X}=\mathscr{O}^{\Omega} / \mathcal{J}_{X}$ we derive, following the ideas in [12, a vector-valued meromorphic $(n, 0)$-form $\omega$ on $X$ such that $\bar{\partial} \omega=0$, i.e, $\omega$ is in $\mathcal{B}_{n}^{X}$. We will show that the product $u \wedge \omega$ has a reasonable meaning when $u$ is in $\mathcal{W}^{X}$. We say that a current $u$ in $\mathcal{W}_{0, *}^{X}$ is in Dom $\bar{\partial}_{X}$ if there is $\phi \in \mathcal{W}_{0, *+1}^{X}$ such that $\bar{\partial}(u \wedge \omega)=\phi \wedge \omega$; we write this equation as $\bar{\partial}_{X} u=\phi$. It turns out that then $\bar{\partial}_{X} u=\phi=\bar{\partial} u$, that also $\phi$ is in Dom $\bar{\partial}_{X}$ and that $\bar{\partial}_{X} \phi=0$. Since $\bar{\partial} \omega=0$ it follows directly that any smooth $(0, *)$-form $u$ is in Dom $\bar{\partial}_{X}$ (and that $\left.\bar{\partial}_{X} u=\bar{\partial} u\right)$. In general, the condition for $u$ being in Dom $\bar{\partial}_{X}$ can be interpreted as a subtle boundary condition on $u$ at $X_{\text {sing }}$, see Lemma 4.1. In case $X$ is a reduced complete intersection, our definition coincides with the definition of $\bar{\partial}$ in [25]. 
Proposition 1.5. We use the notation from Theorem 1.4. Let $\phi$ be in $\mathcal{W}_{0, q}(X)$. If $q \geq 1$, then $\mathscr{K} \phi$ is defined and is in $\mathcal{W}_{0, q-1}\left(X^{\prime}\right)$; if $q=0$, then $\mathscr{P} \phi$ is defined and it is in $\mathscr{O}\left(\Omega^{\prime}\right)$. If $\phi$ is in Dom $\bar{\partial}_{X}$, then the Koppelman formulas (1.2) and (1.3) hold for $z \in X_{\text {reg }}^{\prime}$.

If $\phi \in \mathcal{W}_{0,0}(X)$ and $\bar{\partial}_{X} \phi=0$, thus $\phi \in \mathscr{O}(X)$. If $\phi \in \mathcal{W}_{0, q+1}(X)$, and $\bar{\partial}_{X} \phi=0$, then there is $u \in \mathcal{W}_{0, q}\left(X^{\prime}\right)$ such that $\bar{\partial} u=\phi$ in $X_{r e g}^{\prime}$. However, we do not know whether $\mathscr{K} \phi$, or any other $u$ in $\mathcal{W}_{0, *}^{X}$ that solves $\bar{\partial} u=\phi$ on $X_{\text {reg }}$, is in Dom $\bar{\partial}_{X}$ in general; if $\operatorname{dim} X=1$ then $\mathscr{K} \phi$ is indeed in Dom $\bar{\partial}_{X}$, see Section 9 below. However, we can show that the difference of two such solutions is in fact (strongly) holomorphic if $q=1$ and $\bar{\partial}$-exact on $X_{\text {reg }}$ if $q>1$. By an elaboration of this kind of arguments we can get a global version of Proposition 1.5, see Theorem 1.8 in [10].

Let us mention a few further applications of the Koppelman formulas (1.2) and (1.3). Let $X$ and $X^{\prime}$ be as in Theorem 1.4 and let $\delta(z)$ be the distance to $X_{\text {sing }}$. There is an integer $K$ such that if $\phi \in C_{0, q}^{k}(X), k \geq K+1, q \geq 1$ and $\bar{\partial} \phi=0$ (on $X_{\text {reg }}$ ), then $u=\mathscr{K} \phi$ has meaning, is in $C_{0, q}^{k}\left(X_{\text {reg }}^{\prime}\right), \bar{\partial} u=\phi$ on $X_{\text {reg }}^{\prime}$ and $u(z)=\mathcal{O}\left(\delta(z)^{-M}\right)$ for some integer $M$ not depending on $\phi$, see [10]. Furthermore, if $\phi \in C_{0,0}^{K+1}(X)$ is $\bar{\partial}$-closed on $X_{\text {reg }}$, then $\phi \in \mathscr{O}(X)$; this result was proved in [29] and [40], but our formula gives an explicit holomorphic extension $\mathscr{P} \phi$ to ambient space $\Omega^{\prime}$.

One can incorporate extra weight factors into the Koppelman formulas. In [10] we use this techique to obtain results in the spirit of [21. For instance, we prove that given $M \geq 0$ there is an $M^{\prime} \geq 0$ such that if $\phi$ is a $\bar{\partial}$-closed $(0, q)$-form, $q \geq 1$, on $X_{\text {reg }}$ with $\delta^{-M^{\prime}} \phi \in L^{p}\left(X_{r e g}\right), 1 \leq p \leq \infty$, then there is a $(0, q-1)$-form $u$ on $X_{\text {reg }}^{\prime}$ with $\bar{\partial} u=\phi$ there and $\delta^{-M} u \in L^{p}\left(X_{\text {reg }}\right)$. In [10] we also use extra weight factors to solve the $\bar{\partial}$-equation with compact support. As a consequence we relate extension results to the cohomological dimension $\nu$ of $X$. For instance, if $\nu \geq 2$, then the Hartog's extension phenomenon holds. Moreover, if $\phi$ is a smooth $\bar{\partial}$-closed $(0, q)$-form on $X_{\text {reg }}$ and $q<\nu-1-\operatorname{dim} X_{\text {sing }}$, then there is a smooth solution to the $\bar{\partial}$-equation on $X_{\text {reg }}$; this was proved by Scheja by purely cohomological methods in the early '60s, 39.

Let us also mention a few other works related to the $\bar{\partial}$-equation on complex spaces. In particular, the $L^{2}-\bar{\partial}$-cohomology of (the regular part of) complex spaces (usually with respect to the non-complete metric given by embeddings) has been investigated in, e.g., 31, 30], 33, 34, [18, 32, 36. Also Hölder and $L^{p}$-estimates have been studied, first in [20] and later, using integral formulas, in, e.g., 35], 37].

We have organized the paper as follows. In Section 2 we recall the basic facts from 13 about pseudomeromorphic currents and introduce a slightly more general notion thereof. We also define the sheaf $\mathcal{W}^{X}$. Following the ideas in [12, we introduce in Section 3 a certain residue current $R$ associated to a subvariety of $\mathbb{C}^{N}$ as well as the associated intrinsic structure form $\omega$ on $X$ that is fundamental for the definition of the strong $\bar{\partial}$-operator, $\bar{\partial}_{X}$, in Section 4 and for the construction of the Koppelman formulas. In Section 5 we construct such formulas on a subvariety of a pseudoconvex domain $\Omega \subset \mathbb{C}^{N}$ by using a Koppelman formula in $\Omega$ and "concentrating" it to the subvariety by means of the current $R$. We use these results in Section 6 to obtain intrinsic semi-global Koppelman formulas on an arbitrary pure dimensional reduced complex space. We also prove Proposition 1.5. In Section 7 we define the sheaf $\mathscr{A}^{X}$ 
and prove our main theorem. In Section 8 we provide a few examples that illustrates our construction. As special cases we get back the representation formulas of Stout and Hatziafratis in [41] and 22] for (strongly) holomorphic functions. In the case when $X$ is a complex curve we strengthen our results in Section 9 ,

Acknowledgement: We are grateful to the referee for careful reading and many important comments and suggestions.

\section{The Sheaves $\mathcal{P} \mathcal{M}^{X}$ AND $\mathcal{W}^{X}$}

As in the smooth case, the sheaf $\mathcal{C}_{p, q}^{X}$ of currents of bidegree $(p, q)$ on $X$ is by definition the dual of $\mathscr{D}_{n-q, n-p}^{X}$. Given a local embedding $i: X \hookrightarrow \Omega \subset \mathbb{C}^{N}$, thus, the currents $\mu \in \mathcal{C}_{p, q}^{X}$ precisely correspond, via $\mu \mapsto i_{*} \mu$, to the currents of bidegree $(N-n+p, N-n+q)$ in the ambient space that vanish on all test forms $\xi$ such that $i^{*} \xi=0$ on $X_{\text {reg }}$. If $u$ and $\mu$ are currents on $X$ we say that $\bar{\partial} u=\mu$ if $u$. $\bar{\partial} \xi= \pm \mu . \xi$ for all test forms $\xi$ on $X$. This is equivalent to that $\bar{\partial}\left(i_{*} u\right)=i_{*} \mu$ in the ambient space.

Recall that in one complex variable $x$ one can define the principal value current $1 / x^{m}, m \geq 1$, as the value at $\lambda=0$ of the analytic continuation of $|x|^{2 \lambda} / x^{m}$. Then the residue current $\bar{\partial}\left(1 / x^{m}\right)$ is the value at $\lambda=0$ of $\bar{\partial}|x|^{2 \lambda} / x^{m}$; clearly it has its support at $x=0$. Assume now that $x_{j}$ are holomorphic coordinates on $\mathbb{C}^{N}$. Since we can take tensor products of one-variable currents, we can form the current

$$
\tau=\bar{\partial} \frac{1}{x_{1}^{a_{1}}} \wedge \cdots \wedge \bar{\partial} \frac{1}{x_{r}^{a_{r}}} \wedge \frac{\gamma(x)}{x_{r+1}^{a_{r+1}} \cdots x_{N}^{a_{N}}}
$$

where $a_{1}, \ldots, a_{r}$ are positive integers, $a_{r+1}, \ldots, a_{N}$ are nonnegative integers, and $\gamma$ is a smooth compactly supported form. Such a $\tau$ is called an elementary pseudomeromorphic current. It is commuting in the principal value factors and anti-commuting in the residue factors.

Definition 2.1. We say that a current $\mu$ on $X$ is pseudomeromorphic, $\mu \in \mathcal{P} \mathcal{M}(X)$, if it is locally a finite sum of pushforwards $\pi_{*} \tau=\pi_{*}^{1} \cdots \pi_{*}^{m} \tau$ under maps

$$
X^{m} \stackrel{\pi^{m}}{\longrightarrow} \cdots \stackrel{\pi^{2}}{\longrightarrow} X^{1} \stackrel{\pi^{1}}{\longrightarrow} X^{0}=X
$$

where each $\pi^{j}: X^{j} \rightarrow X^{j-1}$ is either a modification, a simple projection $X^{j-1} \times Z \rightarrow$ $X^{j-1}$, or an open inclusion (i.e., $X^{j}$ is an open subset of $X^{j-1}$ ), and $\tau$ is elementary on $X^{m}$.

Remark 2.2. The notion of pseudomeromorphic currents on a complex manifold was introduced in 13 and the same definition works on our singular space $X$. The definition in 13 is however somewhat more restrictive as it does not allow simple projections. As we will see, all basic properties are preserved also for our class.

It is clear that the pseudomeromorphic currents on $X$ define a subsheaf $\mathcal{P} \mathcal{M}^{X}$ of $\mathcal{C}^{X}$. It is closed under $\bar{\partial}$ and under exterior products with smooth forms since this is true for (finite sums of) elementary currents. Moreover, if $\mu \in \mathcal{P} \mathcal{M}(X)$ and $V \subset X$ is an analytic subvariety, there is a unique pseudomeromorphic current $\mathbf{1}_{X \backslash V} \mu$ on $X$, the so-called standard extension of the natural restriction of $\mu$ to the open set $X \backslash V$. If $h$ is a holomorphic tuple such that $V=\{h=0\}$, then $|h|^{2 \lambda} \mu$ (a priori defined for $\mathfrak{R e} \lambda \gg 0$ ) has a current valued analytic continuation to $\mathfrak{R e} \lambda>-\epsilon$. The value at $\lambda=0$ is precisely the current $\mathbf{1}_{X \backslash V} \mu$, i.e., we have

$$
\mathbf{1}_{X \backslash V} \mu=\left.|h|^{2 \lambda} \mu\right|_{\lambda=0} .
$$


We can also obtain $\mathbf{1}_{X \backslash V} \mu$ as a principal value: If $\chi$ is a smooth approximand of the characteristic function of $[1, \infty)$ on $\mathbb{R}$, then

$$
\mathbf{1}_{X \backslash V} \mu=\lim _{\delta \rightarrow 0^{+}} \chi(|h| / \delta) \mu .
$$

It follows that the current

$$
\mathbf{1}_{V} \mu:=\mu-\mathbf{1}_{X \backslash V} \mu
$$

is in $\mathcal{P} \mathcal{M}^{X}$ and has support on $V$, and in particular that $\mathbf{1}_{V} \mu=\mu$ if $\mu$ has support on $V$. The existence of (2.2) and the independence of $h$ follow from the corresponding statements for elementary currents as in [13], noting that if $\mu=\pi_{*} \tau$, then

$$
|h|^{2 \lambda} \mu=\pi_{*}\left(\left|\pi^{*} h\right|^{2 \lambda} \tau\right)
$$

for $\mathfrak{R e} \lambda \gg 0$. In the same way one can reduce the verification of (2.3) to the case with elementary currents.

These operations satisfy the computation rule

$$
\mathbf{1}_{V} \mathbf{1}_{V^{\prime}} \mu=\mathbf{1}_{V \cap V^{\prime}} \mu .
$$

In fact, if $\mu=\pi_{*} \tau$, then, cf., (2.4),

$$
\mathbf{1}_{V} \mu=\pi_{*}\left(\mathbf{1}_{\pi^{-1}(V)} \tau\right),
$$

and so (2.5) follows from the corresponding statement for the more restricted pseudomeromorphic currents in [13], cf., Remark 2.2.

If $\mu \in \mathcal{P} \mathcal{M}(X)$, then $\mu \otimes 1 \in \mathcal{P} \mathcal{M}(X \times Y)$. To see this, assume that $\mu=\pi_{*} \tau$, where $X^{m} \stackrel{\pi}{\longrightarrow} X$ is a composition of modifications, simple projections, and open inclusions, cf., (2.1), and $\tau$ is elementary on $X^{m}$. Let $Y^{\prime} \stackrel{\pi^{\prime}}{\longrightarrow} Y$ be a modification with $Y^{\prime}$ smooth and let $\left\{\rho_{j}\right\}_{j}$ be a locally finite partition of unity on $Y$. The map $\Pi: X^{m} \times Y^{\prime} \rightarrow X \times Y$ defined by $\Pi(x, y)=\left(\pi(x), \pi^{\prime}(y)\right)$ now is a composition of modifications, simple projections, and open inclusions, $\tau \otimes \Pi^{*} \rho_{j}$ are elementary on $X^{m} \times Y^{\prime}$, and $\mu \otimes 1=\sum_{j} \Pi_{*}\left(\tau \otimes \Pi^{*} \rho_{j}\right)$.

Proposition 2.3. Assume that $\mu \in \mathcal{P} \mathcal{M}(X)$ has support on the subvariety $V \subset X$.

(i) If $\xi \in \mathscr{O}(X)$ is vanishing on $V$, then

$$
\bar{\xi} \mu=0, \quad d \bar{\xi} \wedge \mu=0 .
$$

(ii) (Dimension principle). If $\mu$ has bidegree $(*, p)$ and $\operatorname{codim} V>p$, then $\mu=0$.

The dimension principle is crucial and will be used frequently.

Proof. Assume that $\mu=\pi_{*} \tau$, where $X^{m} \stackrel{\pi}{\longrightarrow} X$ is a composed map as in (2.1) and let $V^{m}:=\pi^{-1} V$. If $\mu$ has support on $V$, then, cf., (2.6),$\mu=\mathbf{1}_{V} \mu=\pi_{*}\left(\mathbf{1}_{V^{m}} \tau\right)$, so $\mu$ is the pushforward of $\tilde{\mu}:=\mathbf{1}_{V^{m}} \tau$, which is pseudomeromorphic in the sense of [13] and has support on $V^{m}$. If $\xi \in \mathscr{O}(X)$ vanishes on $V$, then $\pi^{*} \xi$ vanishes on $V^{m}$ and from [13] we then know that $\pi^{*}(\bar{\xi}) \tilde{\mu}=0$ and $d \pi^{*}(\bar{\xi}) \wedge \tilde{\mu}=0$. Hence, (2.7) holds and so (i) is proved.

Now, choose a local embedding $i: X \hookrightarrow \Omega \subset \mathbb{C}^{N}$. If the hypotheses of (ii) hold, then $i_{*} \mu$ is a current in $\Omega$ of bidegree $(N-n+*, N-n+p)$ that has support on the variety $i(V)$, which has codimension $>N-n+p$. Moreover, $d \bar{\eta} \wedge i_{*} \mu=0$ for every holomorphic function $\eta$ vanishing on $i(V)$ since

$$
d \bar{\eta} \wedge i_{*} \mu=i_{*}\left(d\left(i^{*} \bar{\eta}\right) \wedge \mu\right)=0
$$


in view of part (i). Arguing as in the proofs of Theorem 2.10 and Corollary 2.11 in Chapter III of [17], it now follows that $i_{*} \mu=0$, and hence $\mu=0$.

We say that $\mu \in \mathcal{P} \mathcal{M}(X)$ has the standard extension property, SEP, on $X$ if $\mathbf{1}_{V} \mu=0$ for every subvariety $V \subset X$ of positive codimension.

Definition 2.4 (The sheaf $\mathcal{W}^{X}$ ). Let $X$ be a complex space. We define $\mathcal{W}^{X}$ as the subsheaf of $\mathcal{P} \mathcal{M}^{X}$ of currents with the SEP on $X$.

It is easy to check that

$$
\mathbf{1}_{V}(\phi \wedge \mu)=\phi \wedge \mathbf{1}_{V} \mu
$$

if $\phi$ is a smooth form and $\mu$ is pseudomeromorphic. It follows that the sheaf $\mathcal{W}^{X}$ is closed under multiplication by smooth forms. If $\pi: Y \rightarrow X$ is either a modification or a simple projection, then $\pi_{*}$ maps $\mathcal{W}(Y) \rightarrow \mathcal{W}(X)$. This follows from (2.6) since $\pi^{-1}(V)$ has positive codimension if $V$ has positive codimension.

Let $f$ be holomorphic on $X$. Then $|f|^{2 \lambda} / f$ has an analytic continuation to $\mathfrak{R e} \lambda>-\epsilon$ and the value at $\lambda=0$ is a pseudomeromorphic current, that we denote $1 / f$, which coincides with the function $1 / f$ where $f$ is nonvanishing, see, e.g., [38. We call it the principal value current $1 / f$ associated with the meromorphic function $1 / f$. It follows from the dimension principle that $1 / f$ has the SEP on $X$ and hence is in $\mathcal{W}^{X}$. The product of such a current and a smooth form is called a semi-meromorphic current (or form) in $X$. It will be convenient to introduce a more general notion.

Definition 2.5. We say that a current $\alpha$ on a complex space $X$ is almost semimeromorphic if there is a smooth modification $p: \tilde{X} \rightarrow X$ and a semi-meromorphic current $\tilde{\alpha}$ on $\tilde{X}$ such that $p_{*} \tilde{\alpha}=\alpha$.

Notice that almost semi-meromorphic currents (forms) are in $\mathcal{W}^{X}$. One can also verify that a current $\alpha$ on $X$ is almost semi-meromorphic if and only if there is an analytic set $V \subset X$ of positive codimension such that $\alpha$ is smooth on $X \backslash V$, and a smooth modification $p: \tilde{X} \rightarrow X$, inducing a biholomorphism $\tilde{X} \backslash p^{-1}(V) \rightarrow X \backslash V$, such that $\left(\left.p\right|_{X \backslash V}\right)^{*} \alpha$ extends to a semi-meromorphic current on $\tilde{X}$.

It follows that if $\alpha$ is almost semi-meromorphic in $X$ and $\pi: X^{\prime} \rightarrow X$ is a smooth modification, then the pullback $\pi^{*} \alpha$ is well-defined and again almost semimeromorphic.

Remark 2.6. Let us say that $\alpha$ is locally (almost) semi-meromorphic if for each point there is a neighborhood where $\alpha$ is (almost) semi-meromorphic. For instance, if $f$ is holomorphic in $\Omega \subset \mathbb{C}^{N}$ and $\gamma$ is a test form with support in $\Omega$, then the principal value current $\gamma / f$ is semi-meromorphic in $\Omega$ and locally semi-meromorphic in $\mathbb{C}^{N}$. One can check that a current $\alpha$ on $X$ is locally almost semi-meromorphic if and only if $\alpha$ is pseudomeromorphic in the sense of [13], cf., Remark 2.2, and has the SEP. In fact, fix a point $x$ and suppose that $\alpha$ is a finite sum of currents $\pi_{*} \tau_{\ell}$ as in Remark 2.2 in a neighborhood of $x$. Let $V$ be the union of the images $\pi\left(V_{\ell}\right)$, where for each $\ell, V_{\ell}$ is the affine subspace where the product of the residue factors in $\tau_{\ell}$ has its support. Then $V$ is a germ of an analytic subset of $X$ at $x$ of positive codimension and hence by the SEP, $\mathbf{1}_{V} \alpha=0$. Thus $\alpha$ is the push-forward of elementary currents with no residue factors, and hence it is almost semi-meromorphic at $x$.

\footnotetext{
${ }^{2}$ Somewhat abusively we often identify the meromorphic function and the associated current.
} 
Proposition 2.7. Let $\alpha$ be almost semi-meromorphic on $X$. For each $\mu \in \mathcal{W}(X)$, the current $\alpha \wedge \mu$, a priori defined where $\alpha$ is smooth, has a unique extension to a current in $\mathcal{W}(X)$.

It is natural to let $\alpha \wedge \mu$ denote this extension as well.

Proof. For the uniqueness of an extension of $\alpha \wedge \mu$ in $\mathcal{W}(X)$ we only have to observe that two currents with the SEP on $X$, that coincide outside an analytic set of positive codimension, must be equal everywhere.

Let $V=\{h=0\} \subset X$ be an analytic subset of positive codimension such that $\alpha$ is smooth in $X \backslash V$. We claim that the analytic continuation

$$
\left.|h|^{2 \lambda} \alpha \wedge \mu\right|_{\lambda=0}
$$

exists and defines a current $\nu \in \mathcal{P} \mathcal{M}(X)$. It is clearly a pseudomeromorphic extension of $\alpha \wedge \mu$ from $X \backslash V$ to $X$, and since $\mu$ has the SEP, it follows that $\mathbf{1}_{A} \nu=0$ in $X \backslash V$ for any analytic subset $A \subset X$ with positive codimension. Moreover,

$$
\begin{aligned}
\mathbf{1}_{X \backslash V} \nu & =\left.|h|^{2 \lambda^{\prime}} \nu\right|_{\lambda^{\prime}=0}=\left.\left(\left.|h|^{2 \lambda^{\prime}}|h|^{2 \lambda} \alpha \wedge \mu\right|_{\lambda=0}\right)\right|_{\lambda^{\prime}=0} \\
& =\left.|h|^{2 \lambda^{\prime \prime}} \alpha \wedge \mu\right|_{\lambda^{\prime \prime}=0}=\nu,
\end{aligned}
$$

and in view of (2.5) we can conclude that $\nu$ has the SEP in $X$ and thus is in $\mathcal{W}(X)$.

To prove the claim, let $\mu=\pi_{*} \tau$, where $\pi: X^{m} \rightarrow X$ is a composition of modifications, simple projections, and open inclusions as in (2.1), and $\tau$ is elementary on $X^{m}$. Let $p: \tilde{X} \rightarrow X$ be a smooth modification and let $\tilde{\alpha}$ be a semi-meromorphic current on $\tilde{X}$ such that $p_{*} \tilde{\alpha}=\alpha$. By a standard argument (2.1) can be extended to a commutative diagram

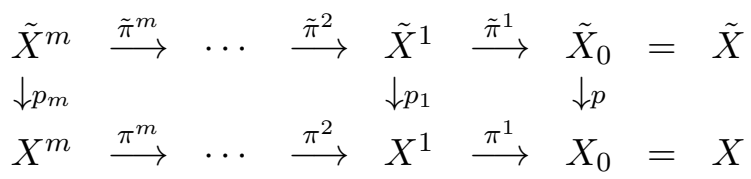

so that each vertical map is a modification and each $\tilde{\pi}^{j}: \tilde{X}^{j} \rightarrow \tilde{X}^{j-1}$ is either a modification, a simple projection, or an open inclusion. Now, let $\tilde{\pi}$ denote the composition of the maps in the first row of (2.10). Then $\hat{\alpha}:=\tilde{\pi}^{*} p^{*} \alpha=\tilde{\pi}^{*} \tilde{\alpha}$ is semi-meromorphic on $\tilde{X}^{m}$ and moreover, there is an elementary (or at least a pseudomeromorphic in the sense of [13]) current $\hat{\tau}$ on $\tilde{X}^{m}$ such that $\left(p_{m}\right)_{*} \hat{\tau}=\tau$. It follows from Proposition 2.1 in [13] that

$$
\lambda \mapsto\left|\tilde{\pi}^{*} p^{*} h\right|^{2 \lambda} \hat{\alpha} \wedge \hat{\tau}
$$

has an analytic continuation to $\mathfrak{R e} \lambda>-\epsilon$ and that the value at $\lambda=0$ defines a pseudomeromorphic current $\hat{\nu}$ on $\tilde{X}^{m}$. Thus, the analytic continuation (2.9) exists and $\nu=\left.|h|^{2 \lambda} \alpha \wedge \mu\right|_{\lambda=0}=p_{*} \tilde{\pi}_{*} \hat{\nu} \in \mathcal{P} \mathcal{M}(X)$.

Example 2.8. Let $W$ be a hypersurface in $X$. We claim that if $\alpha \in \mathcal{P} \mathcal{M}_{k, 0}(X)$ and the restriction $\alpha^{\prime}$ to $X \backslash W$ is holomorphic, then $\alpha$ is meromorphic on $X$. In fact, by assumption, $\alpha^{\prime}$ has a current extension to $X$, so if we have an embedding $i: X \hookrightarrow \Omega$, then the current $i_{*} \alpha^{\prime}$, a priori defined in $\Omega \backslash W$, has a current extension to $\Omega$. By [23. Theorem 1], $\alpha^{\prime}$ has a meromorphic extension $\tilde{\alpha}$ to $X$, and since both $\alpha$ and $\tilde{\alpha}$ are in $\mathcal{P M}_{k, 0}^{X}, \alpha=\tilde{\alpha}$ by the dimension principle.

Let $a$ be a meromorphic form in $\Omega$ such that $\alpha=i^{*} a$. Then $i_{*} \alpha=a \wedge[X]$, where $[X]$ is the Lelong current associated with $X$ in $\Omega$, so $\bar{\partial} \alpha=0$ on $X$ precisely means that $\bar{\partial}(a \wedge[X])=0$ in $\Omega$. This in turn by the definition in [23] means that $\alpha$ is in the 
sheaf (that we denote) $\mathcal{B}_{k}^{X}$ of Barlet-Henkin-Passare holomorphic $(k, 0)$-forms. We conclude that $\mathcal{B}_{k}^{X}$ is the subsheaf of $\bar{\partial}$-closed currents in $\mathcal{P} \mathcal{M}_{k, 0}^{X}$. The sheaves $\mathcal{B}_{k}^{X}$ were defined in this way in [23] but introduced earlier by Barlet, [15], in a different way, see [23, Remark 5].

Example 2.9. Let $a_{1}, \ldots, a_{p}$ be holomorphic on $X$. Following [13] we can inductively define the pseudomeromorphic currents

$$
\mu_{k+1}=\bar{\partial} \frac{1}{a_{k+1}} \wedge \bar{\partial} \frac{1}{a_{k}} \wedge \ldots \wedge \bar{\partial} \frac{1}{a_{1}}:=\left.\frac{\bar{\partial}\left|a_{k+1}\right|^{2 \lambda}}{a_{k+1}} \wedge \bar{\partial} \frac{1}{a_{k}} \wedge \ldots \wedge \bar{\partial} \frac{1}{a_{1}}\right|_{\lambda=0} .
$$

Then $\mu_{p}$ has support on the set $V=\left\{a_{p}=\cdots=a_{1}=0\right\}$. If $X$ is smooth and $\operatorname{codim} V=p$, then $\mu_{p}$ is the so-called Coleff-Herrera product; see [16] and [26] for a thourough discussion of various possible definitions.

\section{The Notion of StRUCTURE Form $\omega$ ON $X$}

To begin with, let $i$ : $X \hookrightarrow \Omega$ be a (reduced) hypersurface in a pseudoconvex domain $\Omega \subset \mathbb{C}^{n+1}$, i.e., $X=\{f=0\}$ where $f$ is holomorphic in $\Omega$ and $d f \neq 0$ on $X_{\text {reg. }}$. If $\omega^{\prime}$ is a meromorphic $(n, 0)$ )-form in $\Omega$ (or in a small neighborhood of $X$ in $\Omega$ ) such that

$$
(d f / 2 \pi i) \wedge \omega^{\prime}=d \zeta_{1} \wedge \ldots \wedge d \zeta_{n+1}
$$

on $X$, then $\omega:=i^{*} \omega^{\prime}$ is a meromorphic form on $X$ that is independent of the choice of $\omega^{\prime}$, and the classical Leray residue formula states that for test forms $\psi$ of bidegree $(0, n-1)$, the principle value integral

$$
\int_{X} \omega \wedge i^{*} \psi
$$

is equal to the action of the residue current $\bar{\partial}(1 / f)$ on the test form $\psi d \zeta_{1} \wedge \ldots \wedge d \zeta_{n+1}$. This equality can be rephrased as

$$
i_{*} \omega=\bar{\partial} \frac{1}{f} \wedge d \zeta_{1} \wedge \ldots \wedge d \zeta_{n+1}
$$

If $\partial f / \partial \zeta_{n+1}$ is not vanishing identically on (any irreducible component of) $X$, one can take, e.g., $\omega^{\prime}=1 /\left(\partial f / \partial \zeta_{n+1}\right) d \zeta_{1} \wedge \ldots \wedge d \zeta_{n}$. Notice that under this assumption on $f$, any meromorphic form on $X$ can be written $h d \zeta_{1} \wedge \ldots \wedge d \zeta_{n}$ for a unique meromorphic function $h$. It follows from (3.2) that $\bar{\partial} \omega=0$ so $\omega$ is in $\mathcal{B}_{n}(X)$, cf., Example 2.8. The form $\omega$ also has the following two properties:

(i) If $\phi$ is a meromorphic function on $X$, then $\phi$ is in $\mathscr{O}^{X}$ if (and only if) $\bar{\partial}(\phi \omega)=0$.

(ii) If $\alpha$ is in $\mathcal{B}_{n}^{X}$ then $\alpha=h \omega$ for some $h$ in $\mathscr{O}^{X}$.

Since any meromorphic $(n, 0)$-form $\alpha$ is $h \omega$ for a unique meromorphic function $h,(i)$ and (ii) are in fact equivalent; for a proof of $(i)$, see, e.g., [23, Remark 3] or below.

For the rest of this section let $i: X \hookrightarrow \Omega \subset \mathbb{C}^{N}$ be a pure $n$-dimensional subvariety of the pseudoconvex domain $\Omega$, and let $p:=N-n$ be its codimension. We will introduce an almost semimeromorphic form $\omega$ on $X$, that satisfies an analogue of (3.2). In a reasonable sense it will also fulfill $(i)$ and $(i i)$. It can be noted that $\omega$ plays a central role in [11. To begin with we look for an adequate generalization of the residue current $\bar{\partial}(1 / f)$. 
If $f$ is any holomorphic function, then a holomorphic function $\phi$ is in the ideal $(f)$ generated by $f$ if and only if $\phi \bar{\partial}(1 / f)=0$. Given any ideal sheaf $\mathcal{J}$ in $\Omega$, in [12] was constructed a residue current $R$ such that

$$
\phi \in \mathcal{J} \text { if and only if } \phi R=0
$$

if $\phi \in \mathscr{O}^{\Omega}$. It is thus reasonable to consider $R$ when $\mathcal{J}=\mathcal{J}_{X}$ is the radical ideal sheaf $\mathcal{J}=\mathcal{J}_{X}$ associated with $X$, so let us first recall its definition. In a slightly smaller set, still denoted $\Omega$, there is a free resolution

$$
0 \rightarrow \mathscr{O}\left(E_{m}\right) \stackrel{f_{m}}{\longrightarrow} \cdots \stackrel{f_{3}}{\longrightarrow} \mathscr{O}\left(E_{2}\right) \stackrel{f_{2}}{\longrightarrow} \mathscr{O}\left(E_{1}\right) \stackrel{f_{1}}{\longrightarrow} \mathscr{O}\left(E_{0}\right)
$$

of the sheaf $\mathscr{O}^{\Omega} / \mathcal{J}_{X}$; here $E_{k}$ are trivial vector bundles over $\Omega$ and $E_{0} \simeq \mathbb{C} \times \Omega$ is the trivial line bundle. This resolution induces a complex of vector bundles

$$
0 \rightarrow E_{m} \stackrel{f_{m}}{\longrightarrow} \cdots \stackrel{f_{3}}{\longrightarrow} E_{2} \stackrel{f_{2}}{\longrightarrow} E_{1} \stackrel{f_{1}}{\longrightarrow} E_{0}
$$

that is pointwise exact outside $X$. Let $X_{k}$ be the set where $f_{k}$ does not have optimal rank. Then

$$
\cdots \subset X_{k+1} \subset X_{k} \subset \cdots \subset X_{p+1} \subset X_{\text {sing }} \subset X_{p}=\cdots=X_{1}=X
$$

these sets are independent of the choice of resolution and thus invariants of the sheaf $\mathscr{F}:=\mathscr{O}^{\Omega} / \mathcal{J}_{X}$. Since $\mathscr{F}$ has pure codimension $p$ (i.e., no embedded prime ideals),

$$
\operatorname{codim} X_{k} \geq k+1, \quad \text { for } \quad k \geq p+1,
$$

see Corollary 20.14 in [19]. There is a free resolution (3.4) if and only if $X_{k}=\emptyset$ for $k>m$. Thus we can always have $m \leq N-1$. The variety is Cohen-Macaulay, i.e., the sheaf $\mathscr{F}$ is Cohen-Macaulay, if and only if $X_{k}=\emptyset$ for $k \geq p+1$. In this case we can thus choose a resolution (3.4) with $m=p$. If we define

$$
X^{0}=X_{\text {sing }}, \quad X^{r}=X_{p+r}, r \geq 1,
$$

then

$$
X^{n-1} \subset \cdots \subset X^{1} \subset X^{0} \subset X, \quad \operatorname{codim} X^{k} \geq k+1 .
$$

The sets $X^{k}$ are independent of the choice of embedding, see [14, Lemma 4.2], and are thus intrinsic subvarieties of the complex space $X$ and reflect the complexity of the singularities of $X$.

Let us now choose Hermitian metrics on the bundles $E_{k}$. We then refer to (3.4) as a Hermitian free resolution of $\mathscr{O}^{X} / \mathcal{J}_{X}$ in $\Omega$. In $\Omega \backslash X_{k}$ we have a well-defined vector bundle morphism $\sigma_{k+1}: E_{k} \rightarrow E_{k+1}$, if we require that $\sigma_{k+1}$ vanishes on $\left(\operatorname{Im} f_{k+1}\right)^{\perp}$, takes values in $\left(\operatorname{Ker} f_{k+1}\right)^{\perp}$ and that $f_{k+1} \sigma_{k+1}$ is the identity on $\operatorname{Im} f_{k+1}$. Following [12] we define the smooth $E_{k}$-valued forms

$$
u_{k}=\left(\bar{\partial} \sigma_{k}\right) \cdots\left(\bar{\partial} \sigma_{2}\right) \sigma_{1}=\sigma_{k}\left(\bar{\partial} \sigma_{k-1}\right) \cdots\left(\bar{\partial} \sigma_{1}\right)
$$

in $\Omega \backslash X$; for the second equality, see [12, (2.3)]. We have that

$$
f_{1} u_{1}=1, \quad f_{k+1} u_{k+1}-\bar{\partial} u_{k}=0, \quad k \geq 1,
$$

in $\Omega \backslash X$. If $f:=\oplus f_{k}$ and $u:=\sum u_{k}$, then these relations can be written economically as $\nabla_{f} u=1$ where $\nabla_{f}:=f-\bar{\partial}$. To make the algebraic machinery work properly one has to introduce a superstructure on the bundle $E=: \oplus E_{k}$ so that vectors in $E_{2 k}$ are even and vectors in $E_{2 k+1}$ are odd, and hence $f, \sigma:=\oplus \sigma_{k}$, and $u:=\sum u_{k}$ 
are odd. For details, see [12]. It turns out that $u$ has a (necessarily unique) almost semi-meromorphic extension $U$ to $\Omega$, and the current $R$ is defined by the relation

$$
\nabla_{f} U=1-R \text {. }
$$

If $F$ is any holomorphic tuple that vanishes on $X$, then

$$
U=\left.|F|^{2 \lambda} u\right|_{\lambda=0}, \quad R=\left.\bar{\partial}|F|^{2 \lambda} \wedge u\right|_{\lambda=0} .
$$

Thus $R$ has support on $X$ and is a sum $\sum R_{k}$, where $R_{k}$ is a pseudomeromorphic $E_{k}$-valued current of bidegree $(0, k)$. It follows from the dimension principle that $R=R_{p}+R_{p+1}+\cdots+R_{N}$. Since we can always choose a resolution that ends at level $N-1$, cf., (3.6), we may assume that $R_{N}=0$. If $X$ is Cohen-Macaulay and $m=p$ in (3.4), then $R=R_{p}$ is $\bar{\partial}$-closed; in general, $R$ is $\nabla_{f}$-closed.

Remark 3.1. If $\mathcal{J}$ is an arbitrary ideal sheaf and $R$ is defined in the same way as above, then (3.3) holds, [12. In case $\mathcal{J}$ is Cohen-Macaulay, one can express this duality in a way that only involves the smooth form $u$ in $\Omega \backslash X$, where $X$ is the zero set of $\mathcal{J}$, see [12, Theorem 4.2]. This result was recently proved algebraically in [28] with no reference to residue calculus and resolution of singularities.

Remark 3.2. In case $\mathcal{J}$ is generated by the single function $f$, then we have the free resolution $0 \rightarrow \mathscr{O} \stackrel{f}{\rightarrow} \mathscr{O} \rightarrow \mathscr{O} /(f) \rightarrow 0$; thus $U$ is just the principal value current $1 / f$ and $R=\bar{\partial}(1 / f)$.

Notice that (3.4) gives rise to the dual Hermitian complex

$$
0 \rightarrow \mathscr{O}\left(E_{0}^{*}\right) \stackrel{f_{1}^{*}}{\rightarrow} \cdots \rightarrow \mathscr{O}\left(E_{p-1}^{*}\right) \stackrel{f_{p}^{*}}{\rightarrow} \mathscr{O}\left(E_{p}^{*}\right) \stackrel{f_{p+1}^{*}}{\longrightarrow} \cdots
$$

Since the sheaf $\operatorname{Ker}\left(\mathscr{O}\left(E_{p}^{*}\right) \stackrel{f_{p+1}^{*}}{\rightarrow} \mathscr{O}\left(E_{p+1}^{*}\right)\right)$ is coherent, there is a (trivial) Hermitian vector bundle $F$ in $\Omega$ and a holomorphic morphism $g: E_{p} \rightarrow F$ such that

$$
\mathscr{O}\left(F^{*}\right) \stackrel{g^{*}}{\rightarrow} \mathscr{O}\left(E_{p}^{*}\right) \stackrel{f_{p+1}^{*}}{\rightarrow} \mathscr{O}\left(E_{p+1}^{*}\right)
$$

is exact. Since $f_{p+1}$ has constant rank outside $X_{p+1}$, also $f_{p+1}^{*}$ has, and it follows that $g$ has as well. Outside $X_{p+1}$ we can thus define the mapping $\sigma_{F}: F \rightarrow E_{p}$ such that $\sigma_{F}=0$ on $(\operatorname{Im} g)^{\perp} \subset F, \sigma_{F} g=I d$ on $(\operatorname{Ker} g)^{\perp}=\left(\operatorname{Im} f_{p+1}\right)^{\perp}$ and $\operatorname{Im} \sigma_{F}$ is orthogonal to Ker $g$. If $m=p$, then we can take $F=E_{p}$ and $g=I d$.

Let $d \zeta:=d \zeta_{1} \wedge \ldots \wedge d \zeta_{N}$. We also introduce the notation

$$
E^{r}:=\left.E_{p+r}\right|_{X}, \quad f^{r}:=\left.f_{p+r}\right|_{X}
$$

so that $f^{r}$ becomes a holomorphic section of $\operatorname{Hom}\left(E^{r}, E^{r-1}\right)$. Notice that for $k \geq 1$, $\alpha^{k}:=i^{*} \bar{\partial} \sigma_{p+k}$ are smooth in $X \backslash X^{k}$.

Proposition 3.3. Let (3.4) be a Hermitian free resolution of $\mathscr{O}^{\Omega} / \mathcal{J}_{X}$ in $\Omega$ and let $R$ be the associated residue current. Then there is a unique almost semi-meromorphic current

$$
\omega=\omega_{0}+\omega_{1}+\cdots+\omega_{n-1}
$$

on $X$, where $\omega_{r}$ has bidegree $(n, r)$ and takes values in $E^{r}$, such that

$$
i_{*} \omega=R \wedge d \zeta .
$$

Moreover,

$$
f^{0} \omega_{0}=0, \quad f^{r} \omega_{r}=\bar{\partial} \omega_{r-1}, r \geq 1, \quad \text { on } X,
$$


and

$$
|\omega|=\mathcal{O}\left(\delta^{-M}\right)
$$

for some $M>0$, where $\delta$ is the distance to $X_{\text {sing }}$.

Assume that (3.11) is exact. The forms $\alpha^{k}, 1 \leq k \leq n-1$, defined and smooth outside $X^{k}$, and $\sigma_{F}$, defined and smooth outside $X^{1}$, extend to almost semimermorphic currents on $X$. There is an F-valued section $\vartheta$ of $\mathcal{B}_{n}^{X}$ such that

$$
\omega_{0}=\sigma_{F} \vartheta \text {. }
$$

Moreover,

$$
\omega_{r}=\alpha_{r} \omega_{r-1}, \quad r \geq 1, \quad \text { on } X .
$$

We say that $\omega$ so obtained is a structure form on $X$. The products in (3.15) and (3.16) are well-defined by Proposition 2.7. Notice that if $X$ is Cohen-Macaulay and $m=p$, then $\omega_{0}$ is an $E^{0}$-valued section of $\mathcal{B}_{n}^{X}$.

Proof. Let $x$ be an arbitrary point on $X_{\text {reg }}$. Since the ideal sheaf $\mathcal{J}_{X}$ is generated by the functions $f_{1}^{j}$ that constitute the map $f_{1}$, cf. (3.4), we can extract holomorphic functions $a_{1} \ldots, a_{p}$ from the $f_{1}^{j}$ 's such that $d a_{1} \wedge \cdots \wedge d a_{p} \neq 0$ at $x$. Possibly after a re-ordering of the variables $\zeta$ in the ambient space, we may assume that $\zeta=$ $\left(\zeta^{\prime}, \zeta^{\prime \prime}\right)=\left(\zeta^{\prime}, \zeta_{1}^{\prime \prime}, \ldots, \zeta_{p}^{\prime \prime}\right)$ and that $A:=\operatorname{det}\left(\partial a / \partial \zeta^{\prime \prime}\right) \neq 0$ at $x$. We also note that $d \zeta^{\prime} \wedge d a_{1} \wedge \cdots \wedge d a_{p}=A d \zeta^{\prime} \wedge d \zeta^{\prime \prime}=A d \zeta$ close to $x$.

Now, $\mathcal{J}_{X}$ is generated by $a=\left(a_{1}, \ldots, a_{p}\right)$ at $x$ and so the Koszul complex generated by the $a_{j}$ provides a minimal resolution of $\mathscr{O}^{\Omega} / \mathcal{J}_{X}$ there. The associated residue current $R^{a}=R_{p}^{a}$ is just the Coleff-Herrera product formed from the tuple $a$, cf., Section 8. The original resolution (3.4) contains the Koszul complex as a direct summand in a neighborhood of $x$ and so it follows from Theorem 4.4 in 12 that

$$
R_{p}=\alpha \bar{\partial} \frac{1}{a_{p}} \wedge \cdots \wedge \bar{\partial} \frac{1}{a_{1}}
$$

where $\alpha$ is a smooth section of $E_{p}$ close to $x$. By the Poincaré-Lelong formula thus

$$
\begin{aligned}
R_{p} \wedge d \zeta & = \pm \alpha \bar{\partial} \frac{1}{a_{p}} \wedge \cdots \wedge \bar{\partial} \frac{1}{a_{1}} \wedge d a_{1} \wedge \cdots \wedge d a_{p} \wedge \frac{d \zeta^{\prime}}{A} \\
& = \pm(2 \pi i)^{p} \alpha \frac{d \zeta^{\prime}}{A} \wedge[X]
\end{aligned}
$$

close to $x$. If $\omega_{0}$ is the pullback of $\pm(2 \pi i)^{p} \alpha d \zeta^{\prime} / A$ to $X_{r e g}$, then the preceding equation means that

$$
R_{p} \wedge d \zeta . \psi=\int_{X} \omega_{0} \wedge i^{*} \psi
$$

where $\psi$ is a test form with support close to $x$. Thus $\omega_{0}$ is determined by $R_{p}$ and so it extends to a global $E_{p}$-valued $(n, 0)$-form on $X_{r e g}$, still denoted $\omega_{0}$. Since $\sigma_{p+1} u_{p}=0$ outside $X_{p+1}$, cf., (3.8), we find that $R_{p}$ and hence $\omega_{0}$ takes values in $\left(\operatorname{Im} f_{p+1}\right)^{\perp} \subset E_{p}$, cf., (3.9) and (3.19). Thus $\omega_{0}=\sigma_{F} g \omega_{0}=\sigma_{F} \vartheta$ where $\vartheta:=g \omega_{0}$. On $X_{\text {reg }}$ we have

$$
i_{*} \bar{\partial} \vartheta=-i_{*} g \bar{\partial} \omega_{0}=-g \bar{\partial} i_{*} \omega_{0}=-g \bar{\partial} R_{p} \wedge d \zeta=-g f_{p+1} R_{p+1} \wedge d \zeta=0
$$

since $g f_{p+1}=0$. Thus $\bar{\partial} \vartheta=0$ and from Example 2.8 we conclude that $\vartheta$ is a section of $\mathcal{B}_{n}^{X}$. 
Let $\mathfrak{a}_{F}$ be the Fitting ideal of $g$, restricted to $X$, i.e., the ideal (on $X$ ) generated by the $r \times r$-minors of $g$, where $r$ is the generic rank of $g$; notice that $g$ has rank $r$ on $X \backslash$ $X^{1}$. Let $\mathfrak{a}_{k}$ be the Fitting ideals of $f^{k}, k=1, \ldots, n-1$. By Hironaka's theorem there is a smooth modification $\tau: \tilde{X} \rightarrow X$ such that all the ideals $\tau^{*} \mathfrak{a}_{F}, \tau^{*} \mathfrak{a}_{1}, \ldots, \tau^{*} \mathfrak{a}_{n-1}$ are principal on $\tilde{X}$. This means that there are holomorphic sections $s_{F}, s_{1}, \ldots, s_{n-1}$ of line bundles on $\tilde{X}$ that generate these ideals. It follows from [12, Lemma 2.1] that $\tau^{*} \sigma_{F}=\beta_{F} / s_{F}$ and $\tau^{*} \sigma^{k}=\beta_{k} / s_{k}, k \geq 1$, where $\beta_{F}$ and $\beta_{k}$ are smooth. Hence, $\tau^{*} \alpha^{k}=\bar{\partial} \beta_{k} / s_{k}$. We conclude that $\sigma_{F}$ as well as $\alpha^{k}$ are almost semi-meromorphic on $X$.

Let us now define $\omega_{r}$ inductively by (3.16). We claim that

$$
i_{*} \omega_{k}=R_{p+k} \wedge d \zeta, \quad k \geq 0 .
$$

If $k=0$ it is just (3.19). Assume (3.20) is proved for $k-1$. It follows from (3.8) and (3.9) that $R_{p+k}=\alpha_{p+k} R_{p+k-1}$ in $\Omega \backslash X_{p+k+1}$. In this set we thus have that

$$
i_{*} \omega_{k}=i_{*} \alpha^{k} \omega_{k-1}=\alpha_{p+k} i_{*} \omega_{k-1}=\alpha_{p+k} R_{k-1} \wedge d \zeta \text {. }
$$

Let $\chi_{\delta}=\chi(|h| / \delta)$, where $h$ is a holomorphic tuple that cuts out $X_{p+k}$, cf., (2.3). Then $i_{*}\left(i^{*} \chi_{\delta} \omega_{k}\right)=\chi_{\delta} R_{k} \wedge d \zeta$. Now $\chi_{\delta} R_{p+k} \rightarrow R_{p+k}$ in view of (3.6) and the dimension principle, and $i^{*} \chi_{\delta} \omega_{k} \rightarrow \omega_{k}$, and hence (3.20) holds in $\Omega$.

The estimate (3.14) follows since it holds for $\Theta$, being a tuple of meromorphic forms on $X$ that are holomorphic on $X_{\text {reg }}$, and for each of $i^{*} \sigma_{F}, \alpha^{1}, \ldots, \alpha^{n-1}$. Finally, (3.13) follows since $(f-\bar{\partial}) R=\nabla_{f} R=0$.

Let $\Theta$ be an $F$-valued meromorphic form in $\Omega$ such that $i^{*} \Theta=\vartheta$. Notice that

$$
\left.\Theta=\gamma_{\Theta}\right\lrcorner d \zeta_{1} \wedge \ldots \wedge d \zeta_{N}
$$

for a (unique) meromorphic section of $F \otimes \Lambda^{p} T^{1,0}(\Omega)$. If $\gamma:=\sigma_{F} \gamma_{\Theta}+\alpha^{1} \sigma_{F} \gamma_{\Theta}+$ $\alpha^{2} \alpha^{1} \sigma_{F} \gamma_{\Theta}+\cdots$ and $\left.\omega^{\prime}:=\gamma\right\lrcorner d \zeta$, thus $\omega=i^{*} \omega^{\prime}$. Since $\left.[X] \wedge \gamma\right\lrcorner d \zeta=[X] \wedge \omega^{\prime}=i_{*} \omega=$ $R \wedge d \zeta$, and $\left.[X] \wedge \gamma\lrcorner d \zeta=(-1)^{p} \gamma\right\lrcorner[X] \wedge d \zeta$ we have

$$
\left.R=(-1)^{p} \gamma\right\lrcorner[X], \quad i_{*} \omega=[X] \wedge \omega^{\prime}=:[X] \wedge \omega .
$$

We will now discuss generalizations of $(i)$ and $(i i)$ above. It is proved in [7] that if $\Phi$ is meromorphic in $\Omega$, then $\phi:=i^{*} \Phi$ is in $\mathscr{O}^{X}$ if and only if $\nabla_{f}(\Phi R)=0$ in $\Omega$. Combining with Proposition 3.3 we get:

$(i)^{\prime}$ If $\phi$ is a meromorphic function on $X$, then $\phi$ is in $\mathscr{O}^{X}$ if and only if $\nabla_{f}(\phi \omega)=0$ on $X$.

Let $\Omega^{k}$ denote the sheaf $\mathscr{O}\left(\Lambda^{k} T_{1,0}^{*}(\Omega)\right)$. Let $\xi d \zeta$ be a section of the sheaf

$$
\mathcal{H o m}_{\mathscr{O}}\left(\mathscr{O}\left(E_{p}\right), \Omega^{N}\right) \simeq \mathscr{O}\left(E_{p}^{*}\right) \otimes_{\mathscr{O}} \Omega^{N}
$$

such that $f_{p+1}^{*} \xi=0$. Then $\bar{\partial}\left(\xi \cdot \omega_{0}\right)=-\xi \cdot \bar{\partial} \omega_{0}=-\xi \cdot f_{p+1} \omega_{1}=f_{p+1}^{*} \xi \cdot \omega_{1}=0$, so that $\xi \cdot \omega_{0}$ is in $\mathcal{B}_{n}^{X}$. The minus signs appear since $f$ is an odd mapping with respect to the superstrucure. Moreover, if $\xi=f_{p}^{*} \eta$ for $\eta \in \mathscr{O}\left(E_{p-1}^{*}\right)$, then $\xi \cdot \omega_{0}=f_{p}^{*} \eta \cdot \omega_{0}=$ $-\eta \cdot f_{p} \omega_{0}=0$. We thus have a sheaf mapping

$$
\mathcal{H}^{p}\left(\mathcal{H o m}\left(\mathscr{O}\left(E_{\bullet}\right), \Omega^{N}\right)\right) \rightarrow \mathcal{B}_{n}^{X}, \quad \xi d \zeta \mapsto \xi \cdot \omega_{0} .
$$

Proposition 3.4. The mapping (3.22) is an isomorphism, and it is independent of the specific choice of resolution, hence establishing an isomorphism

$$
\mathcal{E x t}^{p}\left(\mathscr{O}^{\Omega} / \mathcal{J}_{X}, \Omega^{N}\right) \simeq \mathcal{B}_{n}^{X}
$$


This isomorphism is well-known, cf., [23, Remark 5]. Our contribution is the realization (3.22). Thus $\mathcal{B}_{n}^{X}$ is coherent and we have:

$(\text { ii })^{\prime}$ If $\xi_{1}, \ldots, \xi_{\nu}$ are generators of $\mathcal{H}^{p}\left(\mathcal{H o m}\left(\mathscr{O}\left(E_{\bullet}^{*}\right)\right)\right)$, then $\eta_{\ell}:=\xi_{\ell} \cdot \omega_{0}, \ell=1, \ldots, \nu$, generate the $\mathscr{O}^{X}$-module $\mathcal{B}_{n}^{X}$.

Proof of Proposition 3.4. If $h \in \mathcal{B}_{n}^{X}$, then $i_{*} h=h \wedge[X]$ is a so-called Coleff-Herrera current with respect to $X$ (taking values in the holomorphic vector bundle $\Lambda^{N} T_{1,0}^{*}(\Omega)$ ) that is annihilated by $\mathcal{J}_{X}$, cf., [8]. Thus we have mappings

$$
\mathcal{H}^{p}\left(\mathcal{H o m}\left(\mathscr{O}\left(E_{\bullet}\right), \Omega^{N}\right)\right) \rightarrow \mathcal{B}_{n}^{X} \rightarrow \mathcal{H o m}\left(\mathscr{O}^{\Omega} / \mathcal{J}_{X}, \mathcal{C H}_{X}\right) \otimes_{\mathscr{O}} \Omega^{N},
$$

defined by $\xi d \zeta \mapsto \xi \cdot \omega_{0}$ and $h \mapsto i_{*} h$. The latter mapping is certainly injective. The composed mapping is an isomorphism according to [8, Theorem 1.5]. It follows that both mappings are isomorphisms. From [8, Theorem 1.5] we also know that the composed mapping is independent of the particular Hermitian resolution, and choice of $d \zeta$, and thus induces an isomorphism $\mathcal{E} x t^{p}\left(\mathscr{O}^{\Omega} / \mathcal{J}_{X}, \Omega^{N}\right) \simeq$ $\mathcal{H o m}\left(\mathscr{O}^{\Omega} / \mathcal{J}_{X}, \mathcal{C H}_{X}\right) \otimes_{\mathscr{O}} \Omega^{N}$. Hence the proposition follows.

We conclude with a lemma that roughly speaking says that one can "divide" by $\omega$.

Lemma 3.5. If $\phi$ is a smooth $(n, q)$-form on $X$, then there is a smooth $(0, q)$-form $\phi^{\prime}$ on $X$ with values in $\left(E^{0}\right)^{*}$ such that $\phi=\omega_{0} \wedge \phi^{\prime}$.

Proof. Let $\Phi$ be a smooth extension of $\phi$ to $\Omega$. Since $[X]$ is a Coleff-Herrera current (with values in $\Lambda^{p} T_{1,0}^{*}(\Omega)$ ), it follows from [8, Theorem 1.5 and Example 1] that locally there is a holomorphic $E_{p}^{*}$-valued $(p, 0)$-form $a$ such that $R_{p} \wedge a=[X]$.

By a partition of unity we can find a global smooth $\tilde{a}$ such that $R_{p} \wedge \tilde{a}=[X]$ in $\Omega$. Since $\tilde{a} \wedge \Phi$ has bidegree $(N, q)$, there is an $E_{p}^{*}$-valued smooth $(0, q)$-form $\Phi^{\prime}$ in $\Omega$ such that $\tilde{a} \wedge \Phi=d \zeta \wedge \Phi^{\prime}$. For every test form $\Psi$ in $\Omega$ we now get

$$
\begin{aligned}
\int_{X} \phi \wedge i^{*} \Psi & =[X] .(\Phi \wedge \Psi)=R_{p} \wedge \tilde{a} .(\Phi \wedge \Psi)=R_{p} \wedge d \zeta .\left(\Phi^{\prime} \wedge \Psi\right) \\
& =\int_{X} \omega_{0} \wedge \phi^{\prime} \wedge i^{*} \Psi
\end{aligned}
$$

where $\phi^{\prime}=i^{*} \Phi^{\prime}$. Hence, $\phi=\omega_{0} \wedge \phi^{\prime}$ on $X$.

An algebraic counterpart of the factorization $R_{p} \wedge a=[X]$ appeared in [27] in case $X$ is Cohen-Macaulay; then one can take $a=d f_{1} d f_{2} \cdots d f_{p}$.

\section{The Strong $\bar{\partial}$-Operator ON $X$}

Let $\omega$ be a structure form on $X$, and let $\chi_{\delta}:=\chi(|h| / \delta)$, where $\chi$ is a smooth approximand of the characteristic function of $[1, \infty)$, and $h$ is a holomorphic tuple such that $X_{\text {sing }}=\{h=0\}$. Notice that if $\alpha \in \mathcal{W}(X)$, then

$$
\mathbf{1}_{X_{\text {sing }}} \nabla_{f} \alpha=0 \Longleftrightarrow \mathbf{1}_{X_{\text {sing }}} \bar{\partial} \alpha=0 \Longleftrightarrow \bar{\partial} \chi_{\delta} \wedge \alpha \rightarrow 0, \delta \rightarrow 0
$$

In fact, since $\mathbf{1}_{X_{\text {sing }}} \alpha=0$ and $f$ is smooth we have that $\mathbf{1}_{X_{\text {sing }}} f \alpha=0$; hence the first equivalence follows. For the second one, consider the equality

$$
\bar{\partial}\left(\chi_{\delta} \alpha\right)=\chi_{\delta} \bar{\partial} \alpha+\bar{\partial} \chi_{\delta} \wedge \alpha
$$

Since $\chi_{\delta} \alpha \rightarrow \alpha$ it follows that $\mathbf{1}_{X_{\text {sing }}} \bar{\partial} \alpha=\lim \left(1-\chi_{\delta}\right) \bar{\partial} \alpha=0$ if and only if $\bar{\partial} \chi_{\delta} \wedge \alpha \rightarrow 0$.

Lemma 4.1. Assume that $\mu \in \mathcal{W}(X)$. 
(i) If there is $\tau \in \mathcal{W}(X)$ such that

$$
-\nabla_{f}(\mu \wedge \omega)=\tau \wedge \omega
$$

then $\bar{\partial} \mu=\tau$ and

$$
\bar{\partial} \chi_{\delta} \wedge \mu \wedge \omega \rightarrow 0, \delta \rightarrow 0 .
$$

(ii) If $\bar{\partial} \mu \in \mathcal{W}\left(X_{\text {reg }}\right)$ and (4.3) holds, then there is $\tau \in \mathcal{W}(X)$ such that (4.2) holds.

From Proposition 2.7 we know that $\mu \wedge \omega$ is a well-defined current in $\mathcal{W}(X)$.

Proof. Assume that (4.2) holds. Then $-\mathbf{1}_{X_{\text {sing }}} \nabla_{f}(\mu \wedge \omega)=\mathbf{1}_{X_{\text {sing }}} \tau \wedge \omega=0$, since $\tau \wedge \omega$ is in $\mathcal{W}(X)$. Thus (4.3) holds, in view of (4.1). Since $\omega$ is smooth on $X_{\text {reg }}$ and $\nabla_{f} \omega=0$, (4.2) implies that $\bar{\partial} \mu \wedge \omega_{0}=\tau \wedge \omega_{0}$ on $X_{\text {reg }}$. It follows from Lemma 3.5 that $\bar{\partial} \mu=\tau$ on $X_{\text {reg. }}$. Moreover, from (4.3) and Lemma 3.5 we find that $\bar{\partial} \chi_{\delta} \wedge \mu \rightarrow 0$, so that, cf., (4.1), $\mathbf{1}_{X_{\text {sing }}} \bar{\partial} \mu=0$. It follows that $\bar{\partial} \mu=\mathbf{1}_{X r e g} \bar{\partial} \mu=\tau$. Thus (i) is proved.

If $\bar{\partial} \mu$ has the SEP on $X_{\text {reg }}$, then $\tau:=\mathbf{1}_{X_{r e g}} \bar{\partial} \mu$ has the SEP on $X$ and hence is in $\mathcal{W}(X)$. Since $\omega$ is smooth on $X_{\text {reg }},-\nabla_{f}(\mu \wedge \omega)=\tau \wedge \omega$ there. In view of (4.3) and (4.1), $\mathbf{1}_{X_{\text {sing }}} \nabla_{f}(\mu \wedge \omega)=0$, and since $\nabla_{f}(\mu \wedge \omega)$ has the SEP on $X_{\text {reg }}$ it follows that it has the SEP on $X$, i.e., is in $\mathcal{W}(X)$. Since $\omega$ is smooth on $X_{\text {reg }}$, (4.2) holds on $X_{\text {reg }}$. Since both sides have the SEP, the equality must hold on $X$.

Let $x$ be a point in an arbitrary complex space $X$. By choosing local embeddings $X \hookrightarrow \Omega \subset \mathbb{C}^{N}$ at $x$ and Hermitian free resolutions of $\mathscr{O}^{\Omega} / \mathcal{J}_{X}$ (and choice of coordinates on $\Omega$, cf., (3.12) ) we get the collection $\mathfrak{S}_{x}$ of all structure forms $\omega$ at $x$.

Given $\mu, \tau \in \mathcal{W}_{0, *, x}^{X}$ we say that $\bar{\partial}_{X} \mu=\tau$ at $x$ if (4.2) holds at $x$ for all $\omega \in \mathfrak{S}_{x}$. It follows from Lemma 4.1 that $\bar{\partial}_{X} \mu=\tau$ if and only if $\bar{\partial} \mu=\tau$ and the "boundary condition" (4.3) holds for every $\omega \in \mathfrak{S}_{x}$.

Definition 4.2 (The sheaves $\operatorname{Dom}_{q} \bar{\partial}_{X}$ ). We say that a $(0, q)$-current $\mu$ is a section of $\operatorname{Dom}_{q} \bar{\partial}_{X}$ in the open set $\mathcal{U} \subset X$ if $\mu \in \mathcal{W}_{0, q}(\mathcal{U})$ and there is $\tau \in \mathcal{W}_{0, q+1}(\mathcal{U})$ such that $\bar{\partial}_{X} \mu=\tau$ in $\mathcal{U}$, i.e., $\bar{\partial}_{X} \mu=\tau$ at each point $x \in \mathcal{U}$.

If $\mu \in \mathcal{W}(X)$ is smooth on $X_{\text {reg }}$, then it follows from Lemma 4.1 that $\mu \in \operatorname{Dom}_{q} \bar{\partial}_{X}$ if and only if (4.3) holds at each $x \in X$ for each $\omega \in \mathfrak{S}_{x}$. If $\mu$ is smooth on $X$, then $\bar{\partial}(\mu \wedge \omega)$ has the SEP, and so (4.3) holds for each $\omega$. Thus $\mathscr{E}_{0, q}^{X}$ is a subsheaf of $\operatorname{Dom}_{q} \bar{\partial}_{X}$.

Proposition 4.3. The sheaves $\operatorname{Dom}_{*} \bar{\partial}_{X}$ are $\mathscr{E}_{0, *}^{X}$-modules and

$$
0 \rightarrow \mathscr{O}^{X} \hookrightarrow \operatorname{Dom}_{0} \bar{\partial}_{X} \stackrel{\bar{\partial}}{\longrightarrow} \operatorname{Dom}_{1} \bar{\partial}_{X} \stackrel{\bar{\partial}}{\longrightarrow} \cdots
$$

is a complex. Moreover, the kernel of $\bar{\partial}$ in $\operatorname{Dom}_{0} \bar{\partial}_{X}$ is $\mathscr{O}^{X}$.

When $\operatorname{dim} X=1$ the complex (4.4) is exact, i.e., a fine resolution of $\mathscr{O}^{X}$, see Section 9 below. We do not know whether this is true if $\operatorname{dim} X>1$.

Proof. Assume that $\mu$ is in Dom $\bar{\partial}_{X}$ and that $\omega \in \mathfrak{S}_{x}$. In view of Lemma 4.1, $\mu$ and $\bar{\partial} \mu$ are both in $\mathcal{W}^{X}$ and (4.3) holds. Since $\omega$ is smooth on $X_{\text {reg }}$ and $\nabla_{f} \omega=0$, $\nabla_{f}\left(\bar{\partial} \chi_{\delta} \wedge \mu \wedge \omega\right)=-\bar{\partial} \chi_{\delta} \wedge \bar{\partial} \mu \wedge \omega$. Therefore (4.3), with $\mu$ replaced by $\bar{\partial} \mu$, holds as well and it follows from Lemma 4.1 that $\bar{\partial} \mu \in \operatorname{Dom} \bar{\partial}_{X}$. Moreover, if $\xi$ is smooth it is clear that (4.3) holds with $\mu$ replaced by $\xi \wedge \mu$. Since $\bar{\partial}(\bar{\partial} \mu)=0$ and $\bar{\partial}(\xi \wedge \mu)$ is in $\mathcal{W}^{X}$ we conclude that $\xi \wedge \mu \in \operatorname{Dom} \bar{\partial}_{X}$. 
Now assume $\mu \in \mathcal{W}_{0,0}^{X}$ and (4.2) holds with $\tau=0$. Then $\bar{\partial} \mu=0$ by Lemma 4.1 and hence $\mu$ is holomorphic on $X_{\text {reg }}$, and has a meromorphic extension to $X$, cf., Example 2.8. Thus $\mu \in \mathscr{O}^{X}$ in view of $(i)^{\prime}$ above.

If (4.2) holds at $x$ for a given $\omega \in \mathfrak{S}_{x}$, then in particular, $\bar{\partial}\left(\mu \wedge \omega_{0}\right) \pm \mu f_{p+1} \omega_{1}=$ $\tau \wedge \omega_{0}$. Applying various $\xi \in \mathscr{O}\left(E_{p}^{*}\right)$ with $f_{p+1}^{*} \xi=0$ to this equality we conclude, by Proposition 3.4, that

$$
\bar{\partial}(\mu \wedge \theta)=\tau \wedge \theta, \quad \theta \in \mathcal{B}_{x}^{X} .
$$

If $X$ is Cohen-Macaulay, and (4.2) holds for one $\omega$, then it holds for all $\omega \in \mathfrak{S}_{x}$. In fact we have:

Proposition 4.4. If $X$ is Cohen-Macaulay, then $\mu \in \mathcal{W}(X)$ is in Dom $\bar{\partial}_{X}$ and $\bar{\partial}_{X} \mu=\tau$ if and only if (locally) (4.5) holds.

Proof. It follows from Proposition 3.3 that if $X$ is Cohen-Macaulay at $x \in X$, and thus $X^{1}=\emptyset$, any $\omega \in \mathfrak{S}_{x}$ has the form $a \vartheta$ where $\vartheta$ is (a vector-valued) section of $\mathcal{B}^{X}$ and $a$ is smooth. If $\tau \in \mathcal{W}(X)$ and (4.5) holds, then

$$
\bar{\partial}(\mu \wedge \omega)= \pm \bar{\partial}(a \mu \wedge \vartheta)= \pm \bar{\partial} a \wedge \mu \wedge \vartheta \mp a \bar{\partial}(\mu \wedge \vartheta)= \pm \bar{\partial} a \wedge \mu \wedge \vartheta \mp a \tau \wedge \vartheta .
$$

It follows that $\mathbf{1}_{X_{\text {sing }}} \bar{\partial}(\mu \wedge \omega)=0$ and hence $\mu$ is in Dom $\bar{\partial}_{X}$.

Notice that $\mathcal{W}_{0, n}^{X}=\operatorname{Dom}_{n} \bar{\partial}_{X}$. Assume now that

$$
\operatorname{codim} X^{r} \geq r+\ell, \quad r \geq 0 .
$$

We claim that if $q \leq \ell-2, \mu \in \mathcal{W}_{0, q}^{X}$ and $\bar{\partial} \mu \in \mathcal{W}_{0, q+1}^{X}$, then $\mu \in \operatorname{Dom}_{q} \bar{\partial}_{X}$. To see this, we have to verify that $\mathbf{1}_{X_{\text {sing }}} \bar{\partial}\left(\mu \wedge \omega_{k}\right)=0$ for each $k \geq 0$. For $k=0$ it follows directly by the dimension principle since $\mathbf{1}_{X_{\text {sing }}} \bar{\partial}\left(\mu \wedge \omega_{0}\right)$ has bidegree (at most) $(n, \ell-1)$ and support on $X^{0}$ that has codimension $\ell$. Now, $\omega_{1}=\alpha^{1} \omega_{0}$ and $\alpha^{1}$ is smooth outside $X^{1}$, so $\mathbf{1}_{X_{\text {sing }}} \bar{\partial}\left(\mu \wedge \omega_{1}\right)= \pm \alpha^{1} \mathbf{1}_{X_{\text {sing }}} \bar{\partial}\left(\mu \wedge \omega_{0}\right)=0$ outside $X^{1}$. Thus $\mathbf{1}_{X_{\text {sing }}} \bar{\partial}\left(\mu \wedge \omega_{1}\right)$ has support on $X^{1}$ and hence must vanish by (4.6) and the dimension principle. The claim follows in this way by induction. It follows in particular that $X$ is normal if (4.6) holds for $\ell=2$. One can verify that (4.6) with $\ell=2$ is a way to formulate Serre's conditions $R 1$ and $S 2$ for normality.

\section{Koppelman formulas on $X \subset \Omega$ (The Embedded CONTEXT)}

We first recall the construction of integral formulas in $\left[5\right.$ on an open set $\Omega \subset \mathbb{C}^{N}$. Let $\eta=\left(\eta_{1}, \ldots, \eta_{N}\right)$ be a holomorphic tuple in $\Omega_{\zeta} \times \Omega_{z}$ that generates the ideal associated with the diagonal $\Delta \subset \Omega_{\zeta} \times \Omega_{z}$. For instance one can take $\eta=\zeta-z$. Following the last section in [5] we consider forms in $\Omega_{\zeta} \times \Omega_{z}$ with values in the exterior algebra $\Lambda_{\eta}$ spanned by $T_{0,1}^{*}(\Omega \times \Omega)$ and the $(1,0)$-forms $d \eta_{1}, \ldots, d \eta_{n}$. On such forms interior multiplication $\delta_{\eta}$ with

$$
\eta=2 \pi i \sum_{1}^{N} \eta_{j} \frac{\partial}{\partial \eta_{j}}
$$

is defined. Let $\nabla_{\eta}=\delta_{\eta}-\bar{\partial}$. A smooth section $g=g_{0}+\cdots+g_{N}$ of $\Lambda_{\eta}$, defined for $z \in \Omega^{\prime} \Subset \Omega$ and $\zeta \in \Omega$, such that $\nabla_{\eta} g=0$ and $\left.g_{0}\right|_{\Delta}=1$, lower indices denote degree in $d \eta$, will be called a weight with respect to $z \in \Omega^{\prime}$. Notice that if $g$ and $g^{\prime}$ are weights, then $g \wedge g^{\prime}$ is again a weight. We will use one weight that has compact support in $\Omega$ and one weight which gives a division-interpolation type formula (for 
$z \in \Omega^{\prime}$ ) for the ideal sheaf $\mathcal{J}_{X}$ associated with a subvariety $X \hookrightarrow \Omega$. We first discuss weights with compact support.

Example 5.1 (Weights with compact support). If $\Omega$ is pseudoconvex and $K$ is a holomorphically convex compact subset, then one can find a weight with respect to $z$ in some neighborhood $\Omega^{\prime} \Subset \Omega$ of $K$, depending holomorphically on $z \in \Omega^{\prime}$, that has compact support in $\Omega$, see, e.g., Example 2 in [6]. Here is an explicit choice when $\Omega$ is a neighborhood of the closed unit ball $\overline{\mathbb{B}}, K=\overline{\mathbb{B}}$, and $\eta=\zeta-z$ : Let $\sigma=\bar{\zeta} \cdot d \eta /\left(2 \pi i\left(|\zeta|^{2}-\bar{\zeta} \cdot z\right)\right)$. Then $\delta_{\eta} \sigma=1$ for $\zeta \neq z$ and

$$
\sigma \wedge(\bar{\partial} \sigma)^{k-1}=\frac{1}{(2 \pi i)^{k}} \frac{(\bar{\zeta} \cdot d \eta) \wedge(d \bar{\zeta} \cdot d \eta)^{k-1}}{\left(|\zeta|^{2}-\bar{\zeta} \cdot z\right)^{k}}
$$

If $\chi=\chi(\zeta)$ is a cutoff function that is 1 in a slightly larger ball $\Omega^{\prime}$, then

$$
g=\chi-\bar{\partial} \chi \wedge \frac{\sigma}{\nabla_{\eta} \sigma}=\chi-\bar{\partial} \chi \wedge\left(\sum_{k=1}^{N} \sigma \wedge(\bar{\partial} \sigma)^{k-1}\right) .
$$

is a weight with respect to $z \in \Omega^{\prime}$ with compact support in $\Omega$.

Let $s$ be a smooth $(1,0)$-form in $\Lambda_{\eta}$ such that $|s| \lesssim|\eta|$ and $|\eta|^{2} \lesssim\left|\delta_{\eta} s\right|$; such an $s$ is called admissible. Then $B:=s / \nabla_{\eta} s=\sum_{k} s \wedge(\bar{\partial} s)^{k-1}$ satisfies $\nabla_{\eta} B=1-[\Delta]$, where $[\Delta]$ is the $(N, N)$-current of integration over $\Delta$. If $\eta=\zeta-z$, then $s=\partial|\eta|^{2}$ will do and we refer to the resulting $B$ as the Bochner-Martinelli form. If $g$ is any weight, we have $\nabla_{\eta}(g \wedge B)=g-[\Delta]$, and identifying terms of bidegree $(N, N-1)$ we see that

$$
\bar{\partial}(g \wedge B)_{N}=[\Delta]-g_{N},
$$

which is equivalent to a weighted Koppelman formula in $\Omega$.

We now turn our attention to construction of weights for division-interpolation with respect to the ideal $\mathcal{J}_{X}$. For the rest of this section we assume that $\Omega \subset \mathbb{C}^{N}$ is pseudoconvex and that $X \hookrightarrow \Omega$ is a subvariety. Let us fix global holomorphic frames for the bundles $E_{k}$ in (3.5) over $\Omega$. Then $E_{k} \simeq \mathbb{C}^{\text {rank } E_{k}} \times \Omega$, and the morphisms $f_{k}$ are just matrices of holomorphic functions. One can find, see [6] for explicit choices, $(k-\ell, 0)$-form-valued Hefer morphisms, i.e., matrices $H_{k}^{\ell}: E_{k} \rightarrow E_{\ell}$, depending holomorphically on $z$ and $\zeta$, such that $H_{k}^{k}=I_{E_{k}}$ and

$$
\delta_{\eta} H_{k}^{\ell}=H_{k-1}^{\ell} f_{k}-f_{\ell+1}(z) H_{k}^{\ell+1}, \quad k>\ell,
$$

where $I_{E_{k}}$ is the identity operator on $E_{k}$ and $f$ stands for $f(\zeta)$. For $\mathfrak{R e} \lambda \gg 0$ we put $U^{\lambda}=|F|^{2 \lambda} u$, see Section 3 for the notation, and

$$
R^{\lambda}=\sum_{k=0}^{N} R_{k}^{\lambda}=1-\nabla_{f} U^{\lambda}=1-|F|^{2 \lambda}+\bar{\partial}|F|^{2 \lambda} \wedge u .
$$

Then

$$
\begin{aligned}
g^{\lambda} & :=1-\nabla_{\eta} \sum_{k=1}^{N} H_{k}^{0} U_{k}^{\lambda}=\sum_{k=0}^{N} H_{k}^{0} R_{k}^{\lambda}+f_{1}(z) \sum_{k=1}^{N} H_{k}^{1} U_{k}^{\lambda} \\
& =H R^{\lambda}+f_{1}(z) H U^{\lambda}
\end{aligned}
$$


is a weight that is as smooth as we want if $\mathfrak{R e} \lambda$ is large enough. Let $g$ be any smooth weight with respect to $\Omega^{\prime} \Subset \Omega$ (but not necessarily holomorphic in $z$ ) with compact support in $\Omega_{\zeta}$. Then (5.1) holds with $g$ replaced by $g^{\lambda} \wedge g$. Since $R(z)$ is $\nabla_{f(z)}$-closed we thus get

$$
\begin{aligned}
-\nabla_{f(z)}\left(R(z) \wedge d z \wedge\left(g^{\lambda} \wedge g \wedge B\right)_{N}\right) & =R(z) \wedge d z \wedge[\Delta]- \\
& -R(z) \wedge d z \wedge\left(g^{\lambda} \wedge g\right)_{N} .
\end{aligned}
$$

Notice that the products of currents are well-defined; they are just tensor products since $z$ and $\eta$ are independent variables in $\Omega \times \Omega$. Moreover, since $R(z) f_{1}(z)=0$ we have

$$
\begin{aligned}
-\nabla_{f(z)}\left(R(z) \wedge d z \wedge\left(H R^{\lambda} \wedge g \wedge B\right)_{N}\right) & =R(z) \wedge d z \wedge[\Delta]- \\
& -R(z) \wedge d z \wedge\left(H R^{\lambda} \wedge g\right)_{N} .
\end{aligned}
$$

It follows from (3.12) that (recall that $\Delta \subset \Omega \times \Omega$ is the diagonal)

$$
R(z) \wedge d z \wedge[\Delta]=\iota_{*} \omega,
$$

where $\iota: \Delta^{X} \hookrightarrow \Omega \times \Omega$ is the inclusion of the diagonal $\Delta^{X} \subset X \times X \subset \Omega \times \Omega$. We notice that the analytic continuation to $\lambda=0$ of the last term on the right hand side of (5.2) exists and yields the well-defined current $R(z) \wedge d z \wedge(H R \wedge g)_{N}$ in $\Omega_{\zeta} \times \Omega_{z}^{\prime}$. The existence of the analytic continuation to $\lambda=0$ of the left hand side of (5.2) follows from Proposition 2.1 in 13 since $R(z) \wedge B$ is pseudomoromorphic in $\Omega \times \Omega$. Our Koppelman formulas will follow by letting $\lambda=0$ in (5.2).

To begin with, let us consider (5.2) for $\lambda=0$ in $\left(\Omega \backslash X_{\text {sing }}\right) \times\left(\Omega^{\prime} \backslash X_{\text {sing }}\right)$. In this set we have, by (3.12) and (3.21), that

$$
\begin{aligned}
& \left.R(z) \wedge d z \wedge(H R \wedge g)_{N}= \pm \omega(z) \wedge\left[X_{z}\right] \wedge\left(H(\gamma(\zeta)\lrcorner\left[X_{\zeta}\right]\right) \wedge g\right)_{N} \\
= & \left. \pm \omega(z) \wedge\left[X_{z}\right] \wedge\left[X_{\zeta}\right] \wedge \gamma(\zeta)\right\lrcorner(H \wedge g)_{N}=\omega(z) \wedge\left[X_{z} \times X_{\zeta}\right] \wedge p(\zeta, z),
\end{aligned}
$$

where

$$
\left.p(\zeta, z):= \pm(\gamma(\zeta)\lrcorner(H \wedge g)_{N}\right)_{(n)}
$$

is the term of $\pm \gamma(\zeta)\lrcorner(H \wedge g)_{N}$ of degree $n$ in $d \zeta$; this is the only term of $\left.\pm \gamma(\zeta)\right\lrcorner(H \wedge$ $g)_{N}$ that can contribute in (5.4) since $\omega(z) \wedge\left[X_{z}\right]$ has full degree in the $d z_{j}$. Notice that $p(\zeta, z)$ is almost semi-meromorphic on $X \times X^{\prime}\left(X^{\prime}=X \cap \Omega^{\prime}\right)$ and smooth on $X_{\text {reg }} \times \Omega_{z}^{\prime}$; if $g$ is holomorphic in $z$ then $z \mapsto p(\zeta, z)$ is holomorphic in $\Omega^{\prime}$.

Lemma 5.2. In $\left(\Omega_{\zeta} \backslash X_{\text {sing }}\right) \times\left(\Omega_{z}^{\prime} \backslash X_{\text {sing }}\right)$ we have

$$
\left.R(z) \wedge d z \wedge\left(H R^{\lambda} \wedge g \wedge B\right)_{N}\right|_{\lambda=0}=\left.R(z) \wedge d z \wedge\left(H R \wedge g \wedge|\eta|^{2 \lambda} B\right)_{N}\right|_{\lambda=0} .
$$

Proof. Recall from Section 3 that in $\Omega \backslash X_{\text {sing }}, R$ is a smooth form times $R_{p}$. Notice that

$$
T_{j k}:=\left.R_{p}(z) \wedge R_{k}^{\lambda} \wedge B_{j-k}\right|_{\lambda=0}-\left.R_{p}(z) \wedge R_{k} \wedge|\eta|^{2 \lambda} B_{j-k}\right|_{\lambda=0}, \quad j \leq N .
$$

is a pseudomeromorphic current in $\Omega \times \Omega$ of bidegree $(j-k, p+k+j-k-1)=$ $(j-k, p+j-1)$ that clearly vanishes outside $X_{z}$. It also vanishes outside $\Delta$ since $B$ is smooth there. Thus $T_{j k}$ has support contained in $\Delta^{X} \simeq X$, which has codimension $2 N-n=p+N$ in $\Omega \times \Omega$. Since $p+N>p+j-1$ for $j \leq N$, it follows from the dimension principle that $T_{j k}$ must vanish; in particular, $\left.R_{p}(z) \wedge R_{k}^{\lambda} \wedge B_{j-k}\right|_{\lambda=0}=0$ for $k<p$ since $R_{k}=0$ for $k<p$. We conclude that (5.6) holds in $\left(\Omega \backslash X_{\text {sing }}\right) \times\left(\Omega^{\prime} \backslash X_{\text {sing }}\right)$ since $T_{j k}=0$ there. 
Notice that the right hand side of (5.6) only involves $B_{j}$ with $j \leq n$ since all terms in $H R$ have degree at least $p$ in $d \eta$. If $\Re \mathfrak{R e} \gg 0$ we may replace $g$ by $g \wedge|\eta|^{2 \lambda} B$ in (5.4) and combining with Lemma 5.2 we get

$$
\begin{aligned}
R(z) \wedge & \left.d z \wedge\left(H R^{\lambda} \wedge g \wedge B\right)_{N}\right|_{\lambda=0}=\left.R(z) \wedge d z \wedge\left(H R \wedge g \wedge|\eta|^{2 \lambda} B\right)_{N}\right|_{\lambda=0} \\
& \left.=\omega(z) \wedge[X \times X] \wedge(\gamma(\zeta)\lrcorner\left(H \wedge g \wedge|\eta|^{2 \lambda} B\right)\right)\left._{N}\right|_{\lambda=0} \\
& \left.=\omega(z) \wedge[X \times X] \wedge(\gamma(\zeta)\lrcorner \sum_{j=1}^{n}(H \wedge g)_{N-j} \wedge|\eta|^{2 \lambda} B_{j}\right)\left.\right|_{\lambda=0}
\end{aligned}
$$

in $\left(\Omega \backslash X_{\text {sing }}\right) \times\left(\Omega^{\prime} \backslash X_{\text {sing }}\right)$. Since $B_{j}=\mathcal{O}\left(|\eta|^{-2 j+1}\right)$, we see that $B_{j}$ is locally integrable on $X_{\text {reg }} \times X_{\text {reg }}$ for $j \leq n$. It is thus innocuous to put $\lambda=0$ in the right hand side of (5.7) as long as we restrict our attention to $X_{r e g} \times X_{r e g}^{\prime}$. Notice that the integral kernel

$$
\left.k(\zeta, z):= \pm(\gamma(\zeta)\lrcorner \sum_{j=1}^{n}(H \wedge g)_{N-j} \wedge B_{j}\right)_{(n)}
$$

is almost semi-meromorphic on $X \times X^{\prime}$ and locally integrable on $X_{r e g} \times X_{r e g}^{\prime}$.

In view of (5.2), (5.3), (5.4), (5.7), and (5.8) we have that

$$
-\nabla_{f(z)}(\omega(z) \wedge k(\zeta, z))=\omega \wedge\left[\Delta^{X}\right]-\omega(z) \wedge p(\zeta, z)
$$

in the current sense on $X_{r e g} \times X_{r e g}^{\prime}$. Combined with Lemma 3.5 this gives

Lemma 5.3. With $k(\zeta, z)$ and $p(\zeta, z)$ defined by (5.8) and (5.5) respectively, we have

$$
\bar{\partial} k(\zeta, z)=\left[\Delta^{X}\right]-p(\zeta, z)
$$

in the current sense on $X_{r e g} \times X_{r e g}^{\prime}$.

We can write our integral kernels $p(\zeta, z)$ and $k(\zeta, z)$ in terms of the structure form $\omega$ as follows: Let $F$ be a trivial vector bundle over $\Omega \times \Omega$ with basis elements $\epsilon_{1}, \ldots, \epsilon_{N}$. Now replace each occurrence of $d \eta_{j}$ in $H$ and $g$ by $\epsilon_{j}$ and let $\hat{H}$ and $\hat{g}$ be the forms so obtained. Then

$$
\left.(H \wedge g)_{N}=\epsilon_{N}^{*} \wedge \cdots \wedge \epsilon_{1}^{*}\right\lrcorner\left(d \eta_{1} \wedge \cdots \wedge d \eta_{N} \wedge(\hat{H} \wedge \hat{g})_{N}\right),
$$

where $\left\{\epsilon_{j}^{*}\right\}$ is the dual basis and the lower index $N$ on the right hand side means the term with degree $N$ in the $\epsilon_{j}$. If $C=C(\zeta, z)$ is the invertible holomorphic function defined by $d \eta=C d \zeta+\cdots$, we thus have, cf., (3.21),

$$
\left.p(\zeta, z) \pm C \epsilon_{N}^{*} \wedge \cdots \wedge \epsilon_{1}^{*}\right\lrcorner(\hat{H} \wedge \hat{g})_{N} \wedge \omega(\zeta) .
$$

Similarly, we get that

$$
\left.k(\zeta, z)= \pm C \epsilon_{N}^{*} \wedge \cdots \wedge \epsilon_{1}^{*}\right\lrcorner \sum_{j=1}^{n}(\hat{H} \wedge \hat{g})_{N-j} \wedge \hat{B}_{j} \wedge \omega(\zeta) .
$$




\section{Koppelman formulas on $X$ (in the intrinsic CONTEXT)}

Let $X$ be a reduced complex space of pure dimension $n$. Locally, $X$ can be embedded as a subvariety of a pseudoconvex domain in some $\mathbb{C}^{N}$, so let us, for notational convenience, assume that $X$ can be embedded, $X \hookrightarrow \Omega$, in a pseudoconvex domain $\Omega \subset \mathbb{C}^{N}$. Then, following the previous section, for any $\Omega^{\prime} \Subset \Omega$ we can construct integral kernels $k(\zeta, z)$ and $p(\zeta, z)$ which are almost semi-meromorphic on $X \times X^{\prime}$, where $X^{\prime}=X \cap \Omega^{\prime}$, such that (5.9) and Lemma 5.3 hold. Moreover, $k(\zeta, z)$ and $p(\zeta, z)$ are locally integrable on $X_{\text {reg }} \times X_{\text {reg }}^{\prime}$ and smooth on $X_{r e g} \times \Omega^{\prime}$ respectively.

Now assume that $\mu(\zeta) \in \mathcal{W}_{0, q}(X)$. Since $k(\zeta, z)$ and $p(\zeta, z)$ are almost semimeromorphic, the products $k(\zeta, z) \wedge \mu(\zeta)$ and $p(\zeta, z) \wedge \mu(\zeta)$ are well-defined currents in $\mathcal{W}\left(X_{\zeta} \times X_{z}^{\prime}\right)$ in view of Proposition 2.7. Let $\pi: X_{\zeta} \times X_{z} \rightarrow X_{z}$ be the projection and put $\mathscr{K} \mu(z)=\pi_{*}(k(\zeta, z) \wedge \mu(\zeta))$ and $\mathscr{P} \mu(z)=\pi_{*}(p(\zeta, z) \wedge \mu(\zeta))$. Since $k$ and $p$ have compact support in $\zeta \in \Omega, \mathscr{K} \mu$ and $\mathscr{P} \mu$ are well-defined currents in $\mathcal{W}\left(X_{z}^{\prime}\right)$, and in fact, $\mathscr{P} \mu(z)$ is a smooth function in $\Omega^{\prime}$ since $p(\zeta, z)$ is smooth in $z \in \Omega^{\prime}$; if we choose the weight $g$ to be holomorphic in $z$, then $\mathscr{P} \mu(z)$ is holomorphic in $\Omega^{\prime}$. It is of course natural to write

$$
\mathscr{K} \mu(z)=\int_{X_{\zeta}} k(\zeta, z) \wedge \mu(\zeta), \quad \mathscr{P} \mu(z)=\int_{X_{\zeta}} p(\zeta, z) \wedge \mu(\zeta) .
$$

Lemma 6.1. Let $\mu \in \mathcal{W}_{0, q}(X)$.

(i) If $\mu$ is smooth in a neighborhood of a given point $x \in X_{\text {reg }}^{\prime}$, then $\mathscr{K} \mu(z)$ is smooth in a neighborhood of $x$.

(ii) If $\mu$ vanishes in a neighborhood of $x \in X^{\prime}$, then $\mathscr{K} \mu(z)$ is smooth close to $x$.

Proof. Since $k(\zeta, z)$ is smooth in $z$ close to $x$ if $\zeta$ avoids a neighborhood of $x$, (ii) follows. To see (i) it is enough to assume that $\mu$ is smooth and has compact support close to $x \in X_{\text {reg }}$. Close to the point $(x, x) X \times X$ is a smooth manifold, $\omega(\zeta)$ is smooth, and $\hat{B}_{j} \sim|\zeta-z|^{-2 j+1}$. Thus, (i) follows from the following lemma, cf., the definition (5.8) and (5.11) of $k(\zeta, z)$.

Lemma 6.2. Let $\Phi$ be a non-negative function on $\mathbb{R}_{x}^{d} \times \mathbb{R}_{y}^{d}$ such that $\Phi^{2}$ is smooth and $\Phi \sim|x-y|$. For each integer $m \geq 0$, let $\varphi_{m}$ denote an arbitrary smooth function that is $\mathcal{O}\left(|x-y|^{m}\right)$, and let $\mathcal{E}_{\nu}$ denote a finite sum $\sum_{m \geq 0} \varphi_{m} / \Phi^{\nu+m}$. If $\nu \leq d-1$ and $\xi \in C_{c}^{k}\left(\mathbb{R}^{d}\right)$, then

$$
T \xi(x)=\int_{\mathbb{R}_{y}^{d}} \mathcal{E}_{\nu}(x, y) \xi(y) d y
$$

is in $C^{k}\left(\mathbb{R}^{d}\right)$.

This lemma should be well-known, but for the reader's convenience we sketch a proof.

Sketch of proof. Let $L_{j}=\partial / \partial x_{j}+\partial / \partial y_{j}$. It is readily checked (e.g., by Taylor expanding) that $L_{j} \varphi_{m}=\varphi_{m}$ from which we conclude that $L_{j} \mathcal{E}_{\nu}=\mathcal{E}_{\nu}$. Let

$$
T^{\lambda} \xi(x)=\int_{\mathbb{R}_{y}^{d}}|x-y|^{2 \lambda} \mathcal{E}_{\nu}(x, y) \xi(y) d y .
$$

For $\mathfrak{R e} \lambda>-1 / 2$, it is clear that $T^{\lambda} \xi$ is an analytic $C^{0}\left(\mathbb{R}^{d}\right)$-valued function. Moreover, for $\mathfrak{R e} \lambda>0$, one easily checks by using $L_{j} \mathcal{E}_{\nu}=\mathcal{E}_{\nu}$ that all distributional derivatives of order $\leq k$ of $T^{\lambda} \xi$ are continuous and analytic in $\lambda$ for $\mathfrak{R e} \lambda>-1 / 2$. It follows that $T \xi=T^{0} \xi \in C^{k}\left(\mathbb{R}^{d}\right)$. 
Proposition 6.3. If $\mu$ is a section of $\operatorname{Dom}_{q} \bar{\partial}_{X}$ over $X$, then $\bar{\partial} \mu \in \mathcal{W}_{0, q+1}(X)$ and the current equation

$$
\mu=\bar{\partial} \mathscr{K} \mu+\mathscr{K}(\bar{\partial} \mu)+\mathscr{P} \mu
$$

holds on $X_{\text {reg }}^{\prime}=X_{\text {reg }} \cap \Omega^{\prime}$.

Proof. From Proposition 4.3 it follows that $\bar{\partial} \mu \in \mathcal{W}_{0, q+1}(X)$ and so $\mathscr{K}(\bar{\partial} \mu)$ is a welldefined current in $\mathcal{W}\left(X^{\prime}\right)$. Moreover, from Lemma 5.3 it follows that if $\phi(z)$ is a test form on $X_{r e g}^{\prime}$, then

$$
\phi(\zeta)=\int_{X_{z}} k(\zeta, z) \wedge \bar{\partial} \phi(z)+\bar{\partial}_{\zeta} \int_{X_{z}} k(\zeta, z) \wedge \phi(z)+\int_{X_{z}} p(\zeta, z) \wedge \phi(z)
$$

for $\zeta \in X_{\text {reg }}$. We also see from Lemma 6.1 that all terms in (6.3) are smooth on $X$. If $\mu$ has compact support in $X_{\text {reg }}$, then the proposition follows by duality.

For the general case, let $\chi_{\delta}=\chi(|h| / \delta)$, where $h=h(\zeta)$ is a holomorphic tuple cutting out $X_{\text {sing. }}$. Then the proposition holds for $\chi_{\delta} \mu$. Since $k(\zeta, z) \wedge \mu(\zeta)$ and $p(\zeta, z) \wedge \mu(\zeta)$ has the SEP on $X \times X^{\prime}$, we have that $\mathscr{K}\left(\chi_{\delta} \mu\right) \rightarrow \mathscr{K} \mu$ and $\mathscr{P}\left(\chi_{\delta} \mu\right) \rightarrow$ $\mathscr{P} \mu$. Moreover, $\bar{\partial} \mu \in \mathcal{W}_{0, q+1}(X)$ so $k(\zeta, z) \wedge \bar{\partial} \mu(\zeta)$ has the SEP, which implies that $\mathscr{K}\left(\chi_{\delta} \bar{\partial} \mu\right) \rightarrow \mathscr{K}(\bar{\partial} \mu)$. Hence,

$$
\lim _{\delta \rightarrow 0^{+}} \mathscr{K}\left(\bar{\partial}\left(\chi_{\delta} \mu\right)\right)=\mathscr{K}(\bar{\partial} \mu)+\lim _{\delta \rightarrow 0^{+}} \mathscr{K}\left(\bar{\partial} \chi_{\delta} \wedge \mu\right) .
$$

The singularities of $k(\zeta, z)$ only come from the structure form $\omega(\zeta)$ when $z$ and $\zeta$ "far apart", e.g., for $z$ in a compact subset of $X_{\text {reg }}^{\prime}$ and $\zeta$ close to $X_{\text {sing. From Lemma } 4.1}$ we have that $\bar{\partial} \chi_{\delta} \wedge \mu \wedge \omega \rightarrow 0$ and so $\lim _{\delta \rightarrow 0^{+}} \mathscr{K}\left(\bar{\partial} \chi_{\delta} \wedge \mu\right)=0$ for $z$ in $X_{\text {reg }}^{\prime}$; thus the proposition follows.

Notice that $\mathscr{P} \mu$ in general is smooth. If the weight $g$ is holomorphic in $z$, then $\mathscr{P} \mu$ is holomorphic in $\Omega^{\prime}$ for $q=0$ and 0 for $q \geq 1$. In this case, Proposition 6.3 thus is a homotopy formula for $\bar{\partial}$ on $X_{r e g}^{\prime}$ in the sense that if $\mu$ is in $\operatorname{Dom}_{q} \bar{\partial}_{X}$ on $X$ and $\bar{\partial} \mu=0$, then $\mu$ is holomorphic in $\Omega^{\prime}$ for $q=0$ and $\mu=\bar{\partial} \mathscr{K} \mu$ on $X_{\text {reg }}^{\prime}$ for $q \geq 1$.

Proof of Proposition 1.5. We know that $\mathscr{K} \phi$ is defined and in $\mathcal{W}^{X}$ if $\phi \in \mathcal{W}^{X}$. By choosing the weight $g$ to be holomorphic in $z$, we get that $\mathscr{P} \phi$ is in $\mathscr{O}^{X}$. Moreover, from Proposition 6.3 we have that the Koppelman formulas (1.2) and (1.3) hold on $X_{r e g}^{\prime}$ if, in addition, $\phi$ is in Dom $\bar{\partial}_{X}$.

We do not know whether $\bar{\partial} \mathscr{K} \mu$ is in $\mathcal{W}^{X}$ or not, still less whether $\mathscr{K} \mu$ is in Dom $\bar{\partial}_{X}$ or not in general. However, we shall now see that this is true if $\mu$ is smooth, and more generally if $\mu$ is obtained by a finite number of applications of $\mathscr{K}$ 's. Notice that $\mathscr{K} \mu$ is only defined in the slightly smaller set $X^{\prime}$. Therefore, when we in the following lemma consider products of kernels $\wedge_{j} k_{j}\left(z^{j}, z^{j+1}\right)$, where $\left(z^{1}, \ldots, z^{m}\right)$ are coordinates on $X \times \cdots \times X$, we will assume that $z^{j+1} \mapsto k_{j+1}\left(z^{j+1}, z^{j+2}\right)$ has compact support where $z^{j+1} \mapsto k_{j}\left(z^{j}, z^{j+1}\right)$ is defined.

Lemma 6.4 (Main lemma). Let $k_{j}$ denote kernels (5.8) obtained via local embeddings and arbitrary Hermitian free resolutions of $\mathscr{O}^{\Omega} / \mathcal{J}_{X}$. Let $\left(z^{1}, \ldots, z^{m}\right)$ be coordinates on $X \times \cdots \times X$ and assume that $z^{j+1} \mapsto k_{j+1}\left(z^{j+1}, z^{j+2}\right)$ has compact support where 
$z^{j+1} \mapsto k_{j}\left(z^{j}, z^{j+1}\right)$ is defined. Then, for any $x^{m} \in X$ and any $\omega \in \mathfrak{S}_{x^{m}}$, we have

$$
\lim _{\delta \rightarrow 0^{+}} \bar{\partial} \chi\left(\left|h\left(z^{m}\right)\right| / \delta\right) \wedge \omega\left(z^{m}\right) \wedge \bigwedge_{j=1}^{m-1} k_{j}\left(z^{j}, z^{j+1}\right)=0
$$

in the current sense in a neighborhood of $\left\{x^{m}\right\} \times X \times \cdots \times X$.

Proof. We proceed by induction over $m$. Every $\omega \in \mathfrak{S}_{x^{m}}$ is in $\mathcal{W}^{X}$, so $\chi_{\delta} \omega \rightarrow \omega$ and hence

$$
-\bar{\partial} \chi_{\delta} \wedge \omega=\nabla_{f}\left(\chi_{\delta} \omega\right) \rightarrow \nabla_{f} \omega=0 .
$$

Thus the lemma holds for $m=1$ (i.e., when there are no $k$-kernels). Now consider the case $m+1$. Recall that the limit in (6.4) is a pseudomeromorphic current $T$ in a neighborhood of $\left\{x^{m+1}\right\} \times X \times \cdots \times X$. When $z^{1} \neq z^{2}$, then $k_{1}\left(z^{1}, z^{2}\right)$ is a smooth form times $\omega\left(z^{1}\right)$, cf., (5.11). Thus, outside $z^{1}=z^{2}, T$ is a smooth form times the tensor product of $\omega\left(z^{1}\right)$ and a current of the form (6.4) in the variables $z^{m+1}, \cdots, z^{2}$; the support of $T$ is thus contained in $\left\{z^{1}=z^{2}\right\}$ by the induction hypothesis. For a similar reason the support of $T$ must be contained in $\left\{z^{k}=z^{k+1}\right\}$ and we see that $T$ must have support contained in the diagonal $\Delta=\left\{z^{m+1}=\cdots=z^{1}=0\right\}$. Moreover, the support of $T$ is clearly also contained in $X_{\text {sing }} \times X \times \cdots \times X$. Thus, the support of $T$ is contained in $\left(\Delta^{X}\right)_{\text {sing }} \subset \Delta$, which has dimension (at most) $n-1$ and hence codimension (at least) $(m+1) n-(n-1)=m n+1$.

Now let $T^{0}$ be the component of $T$ obtained from the component $\omega_{0}\left(z^{m+1}\right)$. Then $T^{0}$ has bidegree $(m n, m(n-1)+1)$ since each $k_{j}$ has bidegree $(n, n-1)$. However, since $m \geq 1$, we have $m(n-1)+1<m n+1$ and so $T^{0}=0$ by the dimension principle. Let $T^{1}$ be the component of $T$ obtained from $\omega_{1}\left(z^{m+1}\right)$. Since $\omega_{1}=\alpha^{1} \omega_{0}$ and $\alpha^{1}$ is smooth outside $X^{1}$, it follows from what we have just proved that $T^{1}$ has support contained in $\left(X^{1} \times X \times \cdots \times X\right) \cap \Delta \simeq X^{1}$. This set has codimension at least $m n+1+1$ and $T^{1}$ has bidegree $(*, m(n-1)+1+1)$ so also $T^{1}=0$ by the dimension principle. Proceeding in this way we conclude that $T=0$.

We can now show that Lemma 5.3 holds on $X \times X^{\prime}$.

Proposition 6.5. We have that

$$
-\nabla_{f(z)}(\omega(z) \wedge k(\zeta, z))=\omega \wedge\left[\Delta^{X}\right]-\omega(z) \wedge p(\zeta, z)
$$

in the current sense on $X \times X^{\prime}$.

Proof. Let $\chi_{\delta}=\chi(|h(\zeta)| / \delta)$ and $\chi_{\epsilon}=\chi(|h(z)| / \epsilon)$, where $h$ as before cuts out $X_{\text {sing }}$. From Lemma 5.3 we have that

$$
-\nabla_{f(z)}\left(\chi_{\delta} \chi_{\epsilon} \omega(z) \wedge k(\zeta, z)\right)=\chi_{\delta} \chi_{\epsilon} \omega \wedge\left[\Delta^{X}\right]-\chi_{\delta} \chi_{\epsilon} \omega(z) \wedge p(\zeta, z)+V(\delta, \epsilon),
$$

where

$$
V(\delta, \epsilon)=\bar{\partial} \chi_{\delta} \wedge \chi_{\epsilon} \omega(z) \wedge k(\zeta, z)+\chi_{\delta} \bar{\partial} \chi_{\epsilon} \wedge \omega(z) \wedge k(\zeta, z)
$$

Since $\omega, k, p$, as well as the products $\omega(z) \wedge k(\zeta, z)$ and $\omega(z) \wedge p(\zeta, z)$ all are in $\mathcal{W}(X \times X)$, it is enough to see that $\lim _{\epsilon \rightarrow 0} \lim _{\delta \rightarrow 0} V(\delta, \epsilon)=0$. We have

$$
\lim _{\delta \rightarrow 0} V(\delta, \epsilon)=\lim _{\delta \rightarrow 0} \bar{\partial} \chi_{\delta} \wedge \chi_{\epsilon} \omega(z) \wedge k(\zeta, z)+\bar{\partial} \chi_{\epsilon} \wedge \omega(z) \wedge k(\zeta, z) .
$$

Since $\chi_{\epsilon} \omega(z)$ is smooth and vanishing in a neighborhood of $X_{\text {sing }}, k(\zeta, z)$ is a smooth form times $\omega(\zeta)$, cf., (5.11), on the support of $\bar{\partial} \chi_{\delta}$ if $\delta$ is small enough. Therefore, the first term on the right hand side of (6.5) is 0 by Lemma 6.4 with $m=1$. The second term on the right hand side of (6.5) tends to 0 as $\epsilon \rightarrow 0$, again by Lemma 6.4. 


\section{THE AD HOC SHEAF $\mathscr{A}^{X}$}

We are now ready to define the sheaf $\mathscr{A}^{X}$; it is indeed an ad hoc definition with respect to the Koppelman formulas in the intrinsic context. From the previous two sections we know that we locally (and semi-globally) on $X$ can construct integral kernels $k(\zeta, z)$ and $p(\zeta, z)$, cf., (5.11) and (5.10), and corresponding integral operators $\mathscr{K}$ and $\mathscr{P}$ such that Proposition 6.3 holds.

Definition 7.1. We say that a $(0, q)$-current $\phi$ on an open set $\mathcal{U} \subset X$ is a section of $\mathscr{A}^{X}$ over $\mathcal{U}, \phi \in \mathscr{A}_{q}(\mathcal{U})$, if, for every $x \in \mathcal{U}$, the germ $\phi_{x}$ can be written as a finite sum of terms

$$
\xi_{\nu} \wedge \mathscr{K}_{\nu}\left(\cdots \xi_{2} \wedge \mathscr{K}_{2}\left(\xi_{1} \wedge \mathscr{K}_{1}\left(\xi_{0}\right)\right) \cdots\right)
$$

where $\mathscr{K}_{j}$ are integral operators with kernels $k_{j}(\zeta, z)$ at $x$ of the form defined in Section [5] and $\xi_{j}$ are smooth $(0, *)$-forms at $x$ such that $\xi_{j}$ has compact support in the set where $z \mapsto k_{j}(\zeta, z)$ is defined.

Recall from Section 6 that if $\phi \in \mathcal{W}(\mathcal{U})$ and $\mathscr{K}$ is an integral operator, as defined above, with kernel $k(\zeta, z)$, where $z \mapsto k(\zeta, z)$ is defined in $\mathcal{U}^{\prime} \Subset \mathcal{U}$, then $\mathscr{K} \phi \in \mathcal{W}\left(\mathcal{U}^{\prime}\right)$. Therefore, $\mathscr{A}^{X}$ is a subsheaf of $\mathcal{W}^{X}$ and from Lemma 6.1 it follows that the currents in $\mathscr{A}^{X}$ are smooth on $X_{\text {reg }}$. In view of Lemmas 6.4 and 4.1 we see that $\mathscr{A}^{X}$ is in fact a subsheaf of Dom $\bar{\partial}_{X}$. We also note that if $\phi \in \mathscr{A}_{q}(\mathcal{U})$, then $\mathscr{K} \phi \in \mathscr{A}_{q-1}\left(\mathcal{U}^{\prime}\right)$.

Proof of Theorem 1.2. It is clear that $\mathscr{A}_{q}^{X} \supset \mathscr{E}_{0, q}^{X}$ are fine sheaves satisfying (i) of Theorem 1.2 and we have just noted that also (ii) holds.

We must check condition (iii). We have already seen in Proposition 4.3 that the kernel of $\bar{\partial}$ in $\operatorname{Dom}_{0} \bar{\partial}_{X}$ is $\mathscr{O}^{X}$. Let $\phi$ be a section of $\mathscr{A}_{q}^{X}, q \geq 1$, in a neighborhood of an arbitrary point $x \in X$, and assume that $\bar{\partial} \phi=0$. Since $\mathscr{A}^{X} \subset \operatorname{Dom} \bar{\partial}_{X}$ we also have $\bar{\partial}_{X} \phi=0$. For some neighborhood $\mathcal{U}$ of $x$, by Proposition 6.3, we can find an operator $\mathscr{K}$ such that

$$
\bar{\partial} \mathscr{K} \phi=\phi
$$

in $\mathcal{U}_{\text {reg }}$; here $\mathscr{K}$ corresponds to a weight that is holomorphic in $z$. Since $\phi$ is a section of $\mathscr{A}_{q}^{X}$ we know that $\mathscr{K} \phi$ is a section of $\mathscr{A}_{q-1}^{X}$ and since $\mathscr{A}^{X} \subset$ Dom $\bar{\partial}_{X}$ it follows from Proposition 4.3 that $\bar{\partial} \mathscr{K} \phi$ is in $\mathcal{W}^{X}$. Both sides of (7.1) thus have the SEP and we conclude that (7.1) in fact holds on $\mathcal{U}$.

It remains to prove that $\bar{\partial}$ is a map from $\mathscr{A}^{X}$ to $\mathscr{A}^{X}$. It is sufficient to show that

$$
\bar{\partial}\left(\xi_{\nu} \wedge \mathscr{K}_{\nu}\left(\cdots \xi_{2} \wedge \mathscr{K}_{2}\left(\xi_{1} \wedge \mathscr{K}_{1}\left(\xi_{0}\right)\right) \cdots\right)\right) \in \mathscr{A}^{X},
$$

for any operators $\mathscr{K}_{j}$ (not necessarily corresponding to weights that are holomorphic in $z$ ) and smooth $(0, *)$-forms $\xi_{j}$ with compact support where $\mathscr{K}_{j}\left(\xi_{j-1}\right)$ is defined. We prove (7.2) by induction over $\nu$. The case $\nu=0$ is clear. Assume that (7.2) holds for $\nu=\ell-1$. Let $\mathscr{K}_{j}, j=1, \ldots, \ell$ be any integral operators and $\xi_{j}, j=0, \ldots, \ell$, smooth forms with compact support where $\mathscr{K}_{j}\left(\xi_{j-1}\right)$ are defined. Put $\phi_{\ell-1}=\xi_{\ell-1} \wedge$ $\mathscr{K}_{\ell-1}\left(\cdots \xi_{1} \wedge \mathscr{K}_{1}\left(\xi_{0}\right) \cdots\right)$ and let $\mathcal{U}$ be a sufficiently small neighborhood of $\operatorname{supp} \xi_{\ell}$. By Proposition 6.3 we have that

$$
\phi_{\ell-1}=\mathscr{K}_{\ell}\left(\bar{\partial} \phi_{\ell-1}\right)+\bar{\partial} \mathscr{K}_{\ell} \phi_{\ell-1}+\mathscr{P}_{\ell} \phi_{\ell-1}
$$

in $\mathcal{U}_{\text {reg }}$; notice that $\mathscr{P}_{\ell} \phi_{\ell-1}$ is smooth. From the induction hypothesis we have that $\bar{\partial} \phi_{\ell-1}$ is in $\mathscr{A}^{X}$. Moreover, any $\mathscr{K}$ maps $\mathscr{A}^{X}$ to $\mathscr{A}^{X}$ and since $\mathscr{A}^{X} \subset \operatorname{Dom} \bar{\partial}_{X}$, all terms in (7.3) have the SEP. Hence, (17.3) holds on $\mathcal{U}$ and it follows that $\bar{\partial} \mathscr{K}_{\ell} \phi_{\ell-1}$ is in $\mathscr{A}(\mathcal{U})$. Thus, (7.2) holds for $\nu=\ell$ and the proof is complete. 
Proof of Theorem 1.4. From Section $[$ we have integral operators $\mathscr{K}$ and $\mathscr{P}$ such that $\mathscr{P} \varphi$ is holomorphic in $\Omega^{\prime}$ if $\varphi \in \mathcal{W}_{0,0}(X)$ and 0 if $\varphi \in \mathcal{W}_{0, q}(X), q \geq 1$. Moreover, we noted above that $\mathscr{K}: \mathscr{A}_{q+1}(X) \rightarrow \mathscr{A}_{q}\left(X^{\prime}\right)$ and that $\mathscr{A}^{X}$ is a subsheaf of Dom $\bar{\partial}_{X}$. Let $\phi \in \mathscr{A}_{q}(X), q \geq 1$. By Proposition 6.3 we have that

$$
\phi=\bar{\partial} \mathscr{K} \phi+\mathscr{K}(\bar{\partial} \phi)+\mathscr{P} \phi
$$

on $X_{\text {reg. }}^{\prime}$. Since $\phi$ and $\bar{\partial} \phi$ are in $\mathscr{A}^{X}$, all terms in (7.4) have the SEP, cf., the previous proof. Hence (7.4) holds on $X^{\prime}$ and so Theorem 1.4 follows.

\section{EXAmple With a REDUCED COMPlete INTERSECTION}

Let $a_{1}, \ldots, a_{p} \in \mathscr{O}(\overline{\mathbb{B}})$, where $\mathbb{B} \subset \mathbb{C}^{n}$ is the unit ball, and assume that $X=\left\{a_{1}=\right.$ $\left.\cdots=a_{p}=0\right\} \cap \mathbb{B}$ is a reduced complete intersection, i.e., that $X$ has pure codimension $p$ and $d a_{1} \wedge \cdots \wedge d a_{p} \neq 0$ on $X_{r e g}$. Let $e_{1}, \ldots, e_{p}$ be a holomorphic frame for the trivial bundle $A$ and let $a$ be the section $a=a_{1} e_{1}^{*}+\cdots+a_{p} e_{p}^{*}$ of the dual bundle $A^{*}$, where $\left\{e_{j}^{*}\right\}$ is the dual frame. Put $E_{k}=\Lambda^{k} A$ and let $\delta_{a}: \mathscr{O}\left(E_{\bullet+1}\right) \rightarrow \mathscr{O}\left(E_{\bullet}\right)$ be interior multiplication with $a$. The Koszul complex $\left(\mathscr{O}\left(E_{\bullet}\right), \delta_{a}\right)$ is then a free resolution of $\mathscr{O}^{\Omega} / \mathcal{J}_{X}$, cf., (3.4). It is clear that $s_{a}:=\sum_{j} \bar{a}_{j} e_{j} /|a|^{2}$ is the solution to $\delta_{a} s_{a}=1$, outside $X$, with pointwise minimal norm (with respect to the trivial metric on $A$ ). If we consider all forms as sections of the bundle $\Lambda\left(T^{*}(\Omega) \oplus A\right)$, then we can write (3.8) as $u_{k}=s_{a} \wedge\left(\bar{\partial} s_{a}\right)^{k-1}$. Following [12], cf., (3.9), we get that

$$
R=R_{p}=\left.\bar{\partial}|a|^{2 \lambda} \wedge u_{p}\right|_{\lambda=0}=\bar{\partial} \frac{1}{a_{p}} \wedge \cdots \wedge \bar{\partial} \frac{1}{a_{1}} \wedge e_{1} \wedge \cdots \wedge e_{p}
$$

i.e., $R$ is the classical Coleff-Herrera product (times $e_{1} \wedge \cdots \wedge e_{p}$ ). Let $\omega^{\prime}$ be a smooth $E_{p^{-v a l u e d}}$ form in $\Omega \backslash X_{\text {sing }}$ such that $d a_{1} \wedge \cdots \wedge d a_{p} \wedge \omega^{\prime} /(2 \pi i)^{p}=e \wedge d \zeta$ where $e=e_{1} \wedge \cdots \wedge e_{p}$ and $d \zeta=d \zeta_{1} \wedge \cdots \wedge d \zeta_{N}$. Then the pullback $i^{*} \omega^{\prime}$, where $i: X \hookrightarrow \mathbb{B}$, is unique and meromorphic on $X$. By the Leray residue formula we get that

$$
R \wedge d \zeta=\bar{\partial} \frac{1}{a_{p}} \wedge \cdots \wedge \bar{\partial} \frac{1}{a_{1}} \wedge e \wedge d \zeta=\omega^{\prime} \wedge[X]
$$

and so, cf., (3.12) and (3.21), the structure form associated to $R$ is $\omega:=i^{*} \omega^{\prime}$. If we choose coordinates $\zeta=\left(\zeta^{\prime}, \zeta^{\prime \prime}\right)$ so that $\operatorname{det}\left(\partial a / \partial \zeta^{\prime}\right)$ is generically non-vanishing on $X_{\text {reg }}$, then we can take $\omega^{\prime}=(-2 \pi i)^{p} e \wedge d \zeta^{\prime \prime} / \operatorname{det}\left(\partial a / \partial \zeta^{\prime}\right)$ and the structure form is explicitly given as

$$
\omega=i^{*}\left((-2 \pi i)^{p} e \wedge d \zeta^{\prime \prime} / \operatorname{det}\left(\partial a / \partial \zeta^{\prime}\right)\right)
$$

If we let

$$
\gamma=\frac{(-2 \pi i)^{p}}{\operatorname{det}\left(\partial a / \partial \zeta^{\prime}\right)} e \wedge \frac{\partial}{\partial \zeta_{p}} \wedge \cdots \wedge \frac{\partial}{\partial \zeta_{1}}
$$

then we have $\left.R=(-1)^{p} \gamma\right\lrcorner[X]$, cf., (3.21).

Let $\mu \in \mathcal{W}_{0, q}(X)$ and assume that $\mu$ is smooth on $X_{\text {reg }}$. Then, cf., Section 4, $\mu$ is a section of Dom $\bar{\partial}_{X}$ if and only if $\bar{\partial} \chi_{\delta} \wedge \mu \wedge i^{*}\left(d \zeta^{\prime \prime} / \operatorname{det}\left(\partial a / \partial \zeta^{\prime}\right)\right) \rightarrow 0$ in the current sense as $\delta \rightarrow 0$; here $\chi_{\delta}=\chi(|h| / \delta), \chi$ is a smooth approximand of the characteristic function of $[1, \infty)$ and $h$ cuts out $X_{\text {sing }}$.

To construct integral kernels, cf., Section 5, let $h_{j}$ be $(1,0)$-forms so that $\delta_{\eta} h_{j}=$ $a_{j}(\zeta)-a_{j}(z)$, where $\eta=\zeta-z$. We then have Hefer morphisms $H_{k}^{\ell}$ given as interior multiplication with $\left(\sum h_{j} \wedge e_{j}^{*}\right)^{k-\ell} /(k-\ell)$ !. Let $g$ be the weight from Example 5.1 
and let $B$ be the Bochner-Martinelli form. Then $(H R \wedge g \wedge B)_{N}=H_{p}^{0} R_{p} \wedge(g \wedge B)_{n}$ since $R=R_{p}$ and a straight forward computation shows that

$$
H R=H_{p}^{0} R_{p}=\bar{\partial} \frac{1}{a_{p}} \wedge \cdots \wedge \bar{\partial} \frac{1}{a_{1}} \wedge h_{1} \wedge \cdots \wedge h_{p} .
$$

There is a $\tilde{k}(\zeta, z)=\mathcal{O}\left(|\eta|^{-2 n+1}\right)$ such that

$$
h_{1} \wedge \cdots \wedge h_{p} \wedge(g \wedge B)_{n}=(2 \pi i)^{-p} d \eta \wedge \tilde{k}(\zeta, z)
$$

and so from (5.8) we see that our solution kernel for $\bar{\partial}$ on $X$ is

$$
\left.k(\zeta, z)= \pm(\gamma\lrcorner H_{p}^{0} \wedge(g \wedge B)_{n}\right)_{(n)}= \pm \tilde{k}(\zeta, z) \wedge \frac{d \zeta^{\prime \prime}}{\operatorname{det}\left(\partial a / \partial \zeta^{\prime}\right)} .
$$

Similarly, there is a smooth form $\tilde{p}(\zeta, z)$, depending holomorphically on $z$ if $g$ does, such that

$$
h_{1} \wedge \cdots \wedge h_{p} \wedge(\bar{\zeta} \cdot d \eta) \wedge(d \bar{\zeta} \cdot d \eta)^{n-1}=(2 \pi i)^{-p} d \eta \wedge \tilde{p}(\zeta, z)
$$

and we can compute $p(\zeta, z)$ from (5.5) using $\tilde{p}(\zeta, z)$. We get the representation formula

$$
\phi(z)=\int_{X} \bar{\partial} \chi(\zeta) \wedge \frac{d \zeta^{\prime \prime}}{\operatorname{det}\left(\partial a / \partial \zeta^{\prime}\right)} \wedge \frac{\tilde{p}(\zeta, z)}{\left(|\zeta|^{2}-z \cdot \bar{\zeta}\right)^{n}} \phi(\zeta)
$$

for (strongly) holomorphic functions $\phi$ on $X$. If $X$ intersects $\partial \mathbb{B}$ properly and $X_{\text {sing }}$ avoids $\partial \mathbb{B}$ then we may let $\chi$ tend to the characteristic function for $\mathbb{B}$. The integral (8.2) then becomes an integral over $X \cap \partial \mathbb{B}$ and the resulting representation formula coincides with a formula of Stout [41] and Hatziafratis [22].

Let us consider the cusp $X=\left\{a(z)=z_{1}^{r}-z_{2}^{s}=0\right\} \subset \mathbb{B} \subset \mathbb{C}^{2}$, where $2 \leq r<s$ are relatively prime integers, in more detail. In this case the structure form is the pullback of $-2 \pi i e_{1} \wedge d \zeta_{2} /\left(r \zeta_{1}^{r-1}\right)$ to $X$ and we can take $\gamma(\zeta)=\left(-2 \pi i / r \zeta_{1}^{r-1}\right) \cdot e_{1} \wedge\left(\partial / \partial \zeta_{1}\right)$. The Hefer form is given by

$$
h=h_{1} d \eta_{1}+h_{2} d \eta_{2}=\frac{1}{2 \pi i}\left(\frac{\zeta_{1}^{r}-z_{1}^{r}}{\zeta_{1}-z_{1}} d \eta_{1}+\frac{\zeta_{2}^{s}-z_{2}^{s}}{\zeta_{2}-z_{2}} d \eta_{2}\right)
$$

and we get

$$
h \wedge(g \wedge B)_{1}=h \wedge \chi(\zeta) \frac{\partial|\eta|^{2}}{2 \pi i|\eta|^{2}}=(2 \pi i)^{-1} d \eta_{1} \wedge d \eta_{2} \tilde{k}(\zeta, z)
$$

for a certain function $\tilde{k}(\zeta, z)$. The restriction of this function to $X \times X$ can be computed by applying $\delta_{\eta}$ to (8.3) and noting that $\delta_{\eta} h=a(\zeta)-a(z)=0$ on $X \times X$. One gets that $\tilde{k}(\zeta, z)=\chi(\zeta) h_{1} / \eta_{2}$ on $X \times X$ and so our solution kernel for $\bar{\partial}$ on the cusp is

$$
k(\zeta, z)=\frac{d \zeta_{2}}{r \zeta_{1}^{r-1}} \tilde{k}(\zeta, z)=\frac{\chi(\zeta)}{2 \pi i} \frac{\zeta_{1}^{r}-z_{1}^{r}}{\left(\zeta_{1}-z_{1}\right)\left(\zeta_{2}-z_{2}\right)} \frac{d \zeta_{2}}{r \zeta_{1}^{r-1}} .
$$

Expressed in the parametrization $\tau \mapsto\left(\tau^{s}, \tau^{r}\right)=\left(\zeta_{1}, \zeta_{2}\right)$ our solution operator for $(0,1)$-forms thus becomes

$$
\mathscr{K} \phi(t)=\frac{1}{2 \pi i} \int_{\tau} \chi(\tau) \frac{\tau^{r s}-t^{r s}}{\left(\tau^{s}-t^{s}\right)\left(\tau^{r}-t^{r}\right)} \frac{d \tau}{\tau^{(s-1)(r-1)}} \wedge \phi(\tau) .
$$

One similarly shows that the projection operator $\mathscr{P}$ looks the same but with $\chi$ replaced by $\bar{\partial} \chi$, i.e., the kernel is the same but the solid integral is replaced by a boundary integral. 


\section{The OnE-DIMENSIONAL CASE}

In the case when $X$ is a complex curve we have some further results. In particular, we have a stronger version of Proposition 4.3 .

Proposition 9.1. Let $X$ be a reduced complex curve.

(i) If the complex $0 \rightarrow \mathscr{O}^{X} \hookrightarrow \mathscr{E}_{0,0}^{X} \stackrel{\bar{\partial}}{\longrightarrow} \mathscr{E}_{0,1}^{X} \rightarrow 0$ is exact, then $\mathscr{A}_{*}^{X}=\mathscr{E}_{0, *}^{X}$.

(ii) The complex $0 \rightarrow \mathscr{O}^{X} \hookrightarrow$ Dom $_{0} \bar{\partial}_{X} \stackrel{\bar{\partial}}{\longrightarrow}$ Dom $_{1} \bar{\partial}_{X} \rightarrow 0$ is exact.

Proof. To prove (i), according to Definition [7.1, it is enough to show that $\mathscr{K} \xi$ is smooth for every $\mathscr{K}$ if $\xi$ is a smooth $(0,1)$-form. If $\xi$ is a smooth $(0,1)$-form, there is (locally) a smooth function $\psi$ such that $\bar{\partial} \psi=\xi$. Smooth forms are in $\mathscr{A}^{X}$ and so, cf., the proof of Theorem [1.4, we get that

$$
\mathscr{K} \xi=\mathscr{K}(\bar{\partial} \psi)=\psi-\mathscr{P} \psi
$$

on $X$. Since $\mathscr{P} \psi$ is smooth, $\mathscr{K} \xi$ is indeed smooth on $X$.

From Proposition 4.3 we have that the kernel of $\bar{\partial}$ in $\operatorname{Dom}_{0} \bar{\partial}_{X}$ is $\mathscr{O}^{X}$ so to prove (ii) it remains to see that $\bar{\partial}: \operatorname{Dom}_{0} \bar{\partial}_{X} \rightarrow \operatorname{Dom}_{1} \bar{\partial}_{X}=\mathcal{W}_{0,1}^{X}$ is surjective. We take a minimal local embedding $X \hookrightarrow \mathbb{C}^{N}$ so that $X_{\text {sing }}=\{0\}$ and we let $\mu$ be a section of $\mathcal{W}_{0,1}^{X}$ in a neighborhood of 0 . We choose a Hermitian minimal free resolution of $\mathscr{O}^{X}$ and we get the structure form $\omega=\omega_{0}$; notice that $\mathscr{O}^{X}$ is Cohen-Macaulay since $\operatorname{dim} X=1$. Let $\mathscr{K}$ and $\mathscr{P}$ be integral operators as in Section 6 associated with a weight $g$ which is holomorphic in $z$. From Proposition 6.3 we have that $u_{1}:=\mathscr{K} \mu$ is in $\mathcal{W}_{0,0}^{X}$ and solves $\bar{\partial} u_{1}=\mu$ outside 0 ; we will modify this solution to a solution in $\operatorname{Dom} \bar{\partial}_{X}$.

Let $\pi: \tilde{X} \rightarrow X$ be the normalization of $X$. Then $\tilde{\omega}:=\pi^{*} \omega$ is a meromorphic $(1,0)$-form and from (2.10) we see that there is $\tilde{u}_{1}$ in $\mathcal{W}_{0,0}^{\tilde{X}}$ such that $\pi_{*} \tilde{u}_{1}=u_{1}$. Let $h$ be a holomorphic tuple such that $\{h=0\}=\{0\}$ and put $\chi_{\delta}=\chi(|h| / \delta)$. Then $\nu:=\lim _{\delta \rightarrow 0^{+}} \bar{\partial} \chi_{\delta} \wedge \tilde{u}_{1} \tilde{\omega}$ is a pseudomeromorphic $(1,1)$-current on $\tilde{X}$ with support in the finite set of points $\pi^{-1}(0)$. Let us for simplicity assume that $X$ is irreducible at 0 so that $\tilde{X}$ is connected and $\pi^{-1}(0)$ is just one point $t=0$ for some holomorphic coordinate $t$ on $\tilde{X}$. Then $\nu$ has support at $t=0$ and hence equals a finite linear combination of derivatives of the Dirac mass, $\delta_{0}$, at $t=0$. Moreover, since $\nu$ is pseudomeromorphic, only holomorphic derivatives occur, cf., the first part of the proof of Proposition 2.3, and so we have

$$
\nu=\sum_{0}^{\ell} c_{j}^{\prime} \frac{\partial^{j}}{\partial t^{j}} \delta_{0}=\sum_{0}^{\ell} c_{j} \bar{\partial}\left(\frac{1}{t^{j+1}}\right) \wedge d t, \quad c_{j}^{\prime}, c_{j} \in \mathbb{C} .
$$

Also, since $\tilde{\omega}$ is meromorphic, $\tilde{\omega}=f(t) d t / t^{k}$ for some $k \geq 0$ and some holomorphic function $f$ with $f(0) \neq 0$. The current

$$
\tilde{u}_{2}:=\sum_{j=0}^{\ell} c_{j} \frac{t^{k-j-1}}{f(t)}
$$

is holomorphic for $t \neq 0$ and by construction, $\nu=\bar{\partial}\left(\tilde{u}_{2} \tilde{\omega}\right)$. If $\tilde{u}:=\tilde{u}_{1}-\tilde{u}_{2}$, it is then straightforward to verify that $\bar{\partial} \chi_{\delta} \wedge \tilde{u} \tilde{\omega} \rightarrow 0$ on $\tilde{X}$. Hence, $u:=\pi_{*} \tilde{u}=u_{1}-\pi_{*} \tilde{u}_{2}$ is in $\operatorname{Dom}_{0} \bar{\partial}_{X}$ and solves $\bar{\partial} u=\mu$. 
Notice that once we know that $\bar{\partial}: \operatorname{Dom}_{0} \bar{\partial}_{X} \rightarrow \operatorname{Dom}_{1} \bar{\partial}_{X}=\mathcal{W}_{0,1}^{X}$ is surjective, it is easy to show, using an argument similar to the proof of statement (i) above, that our solution operators for $\bar{\partial}$ indeed produce solutions in $\operatorname{Dom}_{0} \bar{\partial}_{X}$.

Also notice that, in view of Proposition 4.3, it follows from (ii) of Proposition 9.1 that if $H^{1}\left(X, \mathscr{O}^{X}\right)=0$ and $\phi \in \mathcal{W}_{0,1}(X)=\operatorname{Dom}_{1} \bar{\partial}_{X}$, then there is a $\psi \in \mathcal{W}_{0,0}(X)$ such that $\bar{\partial}_{X} \psi=\phi$ on $X$.

\section{REFERENCES}

[1] V. Ancona, B. Gaveau: Differential forms and resolutions on certain analytic spaces. I. Irreducible exceptional divisor. Bull. Sci. Math., 116(3) (1992), 307-324.

[2] V. Ancona, B. Gaveau: Differential forms and resolutions on certain analytic spaces. II. Flat resolutions. Canad. J. Math., 44(4) (1992), 728-749.

[3] V. Ancona, B. Gaveau: Differential forms and resolutions on certain analytic spaces. III. Spectral resolution. Ann. Math. Pure Appl.(4), 166 (1994), 175-202.

[4] V. Ancona, B. Gaveau: Bochner-Martinelli formulas on singular complex spaces. Internat. J. Math., 21(2) (2010), 225-253.

[5] M. Andersson: Integral representation with weights I. Math. Ann., 326 (2003), 1-18.

[6] M. Andersson: Integral representation with weights II, division and interpolation formulas. Math. Z., 254 (2006), 315-332.

[7] M. Andersson: A residue criterion for strong holomorphicity. Ark. Mat., 48(1) (2010), 1-15.

[8] M. Andersson: Coleff-Herrera currents, duality, and Noetherian operators. Bull. Soc. Math. France, to appear. Available at arXiv:0902.3064 math.CV].

[9] M. Andersson, H. Samuelsson: Koppelman formulas and the $\bar{\partial}$-equation on an analytic space. arXiv:0801.0710

[10] M. Andersson, H. Samuelsson: Koppelman formulas and the $\bar{\partial}$-equation on an analytic space. Journal of functional analysis, 261 (2011), 777-802.

[11] M. Andersson, H. Samuelsson, J. Sznajdman: On the Briançon-Skoda theorem on a singular variety. Ann. Inst. Fourier (Grenoble), 60(2) (2010), 417-432.

[12] M. Andersson, E. Wulcan: Residue currents with prescribed annihilator ideals. Ann. Sci. École Norm. Sup., 40 (2007), 985-1007.

[13] M. Andersson, E. Wulcan: Decomposition of residue currents. J. Reine Angew. Math., 638 (2010), 103-118.

[14] M. Andersson, E. Wulcan: On the effective membership problem on singular varieties. arXiv:110\%.0388.

[15] D. BARlet: Le faisceau $\omega_{X}$ sur un espace analytique $X$ de dimension pure. Fonctions de plusieurs variables complexes, III (Sém. François Norguet, 1975-1977) 187-204, Lecture Notes in Math., 670, Springer, Berlin, 1978.

[16] J-E. Björk, H. SAmuelsson: Regularizations of residue currents. J. Reine Angew. Math., 640 (2010), 101-115.

[17] J.-P. Demailly: Complex analytic and algebraic geometry. Monograph, Grenoble. Available

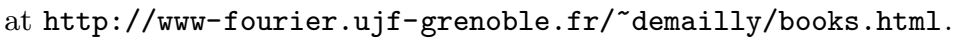

[18] K. Diederich, J. E. Fornæss, S. Vassiliadou: Local $L^{2}$ results for $\bar{\partial}$ on a singular surface. Math. Scand., 92 (2003), 269-294.

[19] D. Eisenbud: Commutative algebra. With a view toward algebraic geometry. Graduate Texts in Mathematics, 150. Springer-Verlag, New-York, 1995.

[20] J. E. Fornæss, E. A. Gavosto: The Cauchy-Riemann equation on singular spaces. Duke Math. J., 93 (1998), 453-477.

[21] J. E. Fornæss, N. Øvrelid, S. Vassiliadou: Semiglobal results for $\bar{\partial}$ on a complex space with arbitrary singularities. Proc. Am. Math. Soc., 133(8) (2005), 2377-2386.

[22] T. E. Hatziafratis: Integral representation formulas formulas on analytic varieties. Pacific J. Math., 123(1) (1986), 71-91.

[23] G. Henkin, M. Passare: Abelian differentials on singular varieties and variations on a theorem of Lie-Griffiths. Invent. Math., 135(2) (1999), 297-328.

[24] G. Henkin, P. Polyakov: The Grothendieck-Dolbeault lemma for complete intersections. $C$. R. Acad. Sci. Paris Sér I Math, 308(13) (1989), 405-409. 
[25] G. Henkin, P. Polyakov: Residual d-bar-cohomology and the complex Radon transform on subvarieties of CPn. arXiv:1012.4438

[26] R. LÄrkäNG, H. SAmuelsson: Various approaches to products of residue currents. Preprint Göteborg, 2010. Available at arXiv:1005.2056 math.CV].

[27] M. Lejeune-Jalabert: Remarque sur la classe fondamentale d'un cycle. C. R. Acad. Sci. Paris Sér. I Math. 292 (1981), 801-804.

[28] J. Lundqvist: A local Grothendieck duality theorem for Cohen-Macaulay ideals. Preprint Stockholm 2011.

[29] B. Malgrange: Sur les fonctions différentiables et les ensembles analytiques. Bull. Soc. Math. France, 91 (1963), 113-127.

[30] M. Nagase: Remarks on the $L^{2}$-Dolbeault cohomology groups of singular algebraic surfaces and curves. Publ. Res. Inst. Math. Sci., 26(5) (1990), 867-883.

[31] T. OhsAwa: Hodge spectral sequences on compact Kähler spaces. Publ. Res. Inst. Math. Sci., 23(2) (1987), 265-274.

[32] N. Øvrelid, S. Vassiliadou: Some $L^{2}$ results for $\bar{\partial}$ on projective varieties with general singularities. Amer. J. Math., 131 (2009), 129-151.

[33] W. Pardon, M. Stern: $L^{2}-\bar{\partial}$-cohomology of complex projective varieties. J. Amer. Math. Soc., 4(3) (1991), 603-621.

[34] W. Pardon, M. Stern: Pure Hodge structure on the $L^{2}$-cohomology of varieties with isolated singularities. J. Reine Angew. Math., 533 (2001), 55-80.

[35] J. Ruppenthal: Zur Regularität der Cauchy-Riemannschen Differentialgleichungen auf komlexen Räumen. Diplomarbeit, Bonn 2003, and dissertation published in Bonner Math. Schr., 380 (2006).

[36] J. Ruppenthal: $L^{2}$-theory for the $\bar{\partial}$-operator on compact complex spaces. ESI-Preprint 2202, available at arXiv:1004.0396 math.CV].

[37] J. Ruppenthal, E. S. Zeron: An explicit $\bar{\partial}$-integration formula for weighted homogeneous varieties II, forms of higher degree. Mich. Math. J., 59 (2010), 283-295.

[38] H. Samuelsson: Analytic continuation of residue currents. Ark. Mat., 47(1) (2009), 127-141.

[39] G. ScheJA: Riemannsche Hebbarkeitssätze für Cohomologieklassen. Math. Ann., 144 (1961), 345-360.

[40] K. Spallek: Über Singularitäten analytischer Mengen. Math. Ann., 172 (1967), 249-268.

[41] E. L. Stout: An integral formula for holomorphic functions on strictly pseudoconvex hypersurfaces. Duke Math. J., 42 (1975), 347-356.

M. Andersson, Department of Mathematical Sciences, Division of Mathematics, University of Gothenburg and Chalmers University of Technology, SE-412 96 Göteborg, SWEDEN

E-mail address: matsa@chalmers.se

H. Samuelsson, Department of Mathematical Sciences, Division of Mathematics, University of Gothenburg and Chalmers University of Technology, SE-412 96 Göteborg, SWEDEN

E-mail address: haakan.samuelsson@gmail.com 